\title{
MONGE-AMPÈRE EXHAUSTIONS OF ALMOST HOMOGENEOUS MANIFOLDS
}

\author{
MORRIS KALKA, GIORGIO PATRIZIO AND ANDREA SPIRO
}

Dedicated to Ngaiming Mok for his sixtieth birthday

\begin{abstract}
We consider three fundamental classes of compact almost homogenous manifolds and show that the complements of singular complex orbits in such manifolds are endowed with plurisubharmonic exhaustions satisfying complex homogeneous MongeAmpère equations. This extends to a new family of mixed type examples various classical results on parabolic spaces and complexifications of symmetric spaces. Rigidity results on complex spaces modeled on such new examples are given.
\end{abstract}

\section{INTRODUCTION}

Plurisubharmonic exhaustions satisfying the complex homogeneous Monge-Ampère equation on a complex space appear naturally in many contexts. Probably the first time they have been extensively considered was in Value Distribution Theory on affine algebraic varieties or, more generally, on parabolic spaces ([11, 33]). When the exhaustions satisfy the complex homogeneous Monge-Ampère equation with the least possible degeneracy - the strictly parabolic case in the terminology of Stoll - a natural foliation is associated to the exhaustion, namely the collection of complex curves that are tangent to the annihilators of the Levi form. The very nice behavior of these exhaustions in this case and the analogy with the case of Riemann surfaces, suggested Stoll that these might be instrumental for the characterization of special complex manifolds such as $\mathbb{C}^{n}$, the unit ball $\mathbb{B}^{n}$, bounded complete circular domains or affine cones. It is well known that this is indeed the case (see for instance [34, 8, 35, 23, 36, 26]). In all these instances the minimal set of the exhaustion is always a point or, after blowing up to resolve singularities, a compact projective manifold. The exhaustion has always a logarithmic type of singularity along such minimal set. In these examples, there is a sharp difference of behaviors, depending on whether or not the plurisubharmonic exhaustions, satisfying the complex homogeneous Monge-Ampère equation, is bounded from above. If the exhaustion is unbounded, the associated foliation is necessarily holomorphic, the holomorphic type of the manifold is fixed and the only allowed deformations are rescalings of the exhaustion in the direction of the leaves of the foliation $([8,26])$. On the other hand, when the exhaustion is bounded above, there is a very rich class of non biholomorphically inequivalent examples which are suitable deformation of the unit ball $\mathbb{B}^{n} \subset \mathbb{C}^{n}$ - in fact an infinite dimensional class, see [16, 17, 5, 24] - and, up to rescaling, the plurisubharmonic exhaustions that satisfy the complex homogeneous Monge-Ampère equation are pluricomplex Green functions.

2010 Mathematics Subject Classification. 32U10, 32W20, 32M12.

Key words and phrases. Monge-Ampère Equations, Almost Homogenous Manifolds, Plurisuharmonic Exhaustions, Deformation of Complex Structures.

Acknowledgments. This research was partially supported by the Project MIUR "Real and Complex Manifolds: Geometry, Topology and Harmonic Analysis" and by GNSAGA of INdAM. 
In a rather different development, plurisubharmonic exhaustions satisfying the complex homogeneous Monge-Ampère equation occur as a natural byproduct of the construction of intrinsic complexifications of real analytic compact Riemannian manifolds, the so called Grauert Tubes. They are tubular neighborhoods of each such manifold in its tangent bundle - possibly the entire tangent bundle - equipped with an adapted complex structure with the property that the differentials of parameterizations of geodesics of the manifold are holomorphic embeddings into the Grauert tube of strip shaped neighborhoods of the real line in $\mathbb{C}$ (see e.g. [13, 18, 27]; see also [10] for generalizations to the Finsler case). Here the real analytic Riemannian manifold sits in the Grauert tube as a top dimensional totally real submanifold, and it is the minimal set of the norm function on the tangent bundle determined by the Riemannian metric. Considering the above described adapted complex structure, the norm function turns out to be a plurisubharmonic exhaustion satisfying the complex homogeneous Monge-Ampère equation and exhibiting a "square root" type of singularity along its minimal set, that is the real analytic Riemannian manifold on which the Grauert tube has been built. Note that, in this case, once the size of the tubular neighborhood is fixed, whether finite or - when possible - infinite, there is a strongly rigid behavior: the isometric type of the minimal set of the exhaustion completely determines the complex structure of the Grauert tube.

In this paper we consider a large class of almost homogeneous complex manifolds with cohomogeneity one actions, that is complex manifolds $M$ with a real Lie group $G$ of biholomorphisms acting with real hypersurfaces as principal orbits. Almost homogeneous complex manifolds have been extensively studied and classified (see [14, 15, 1] and, for the strictly related topic of the classification of compact homogeneous CR manifolds, [2, 3]). Under the additional assumption that all principal $G$-orbits are strongly pseudoconvex hypersurfaces and that the manifold has vanishing first Betti number, it is possible to fully describe all compact almost $G^{\mathbb{C}}$-homogeneous manifolds with cohomogeneity one $G$-actions of strongly pseudoconvex principal orbits ([29]). It turns out that, up to blow ups, there are three types of such manifolds (see $\$ 3.2$ below for details):

- Type 1: Almost homogeneous manifolds with two compact complex manifolds as exceptional orbits; they are all $\mathbb{C} P^{1}$ bundles over a flag manifold.

- Type 2: Almost homogeneous manifolds with one compact complex exceptional orbit and one totally real; they are the compactifications of the Morimoto-Nagano manifolds, i.e. the compactifications of the standard complexifications of compact symmetric spaces of rank one (CROSS).

- Type 3: A finite list of exceptional almost homogeneous manifolds with one compact complex exceptional orbit and one compact exceptional orbit of mixed real/complex type; the latter is a bundle over a flag manifold with fiber which is either a sphere or a real projective space of specified dimension.

Each such manifold $M$ has always two singular $G$-orbits and at least one of them is complex. If $S \subset M$ is such a complex orbit, then on $M_{o}:=M \backslash S$ there exists (see Theorem 3.2 for precise statement) a $\mathcal{C}^{\infty}$ exhaustion $\tau: M_{o} \rightarrow[0, \infty)$, such that $\{\tau=0\}$ is exactly the other singular $G$-orbit $S_{o}$ of $M$ and whose restriction to $M_{o} \backslash\{\tau=0\}=M_{o} \backslash S_{o}$ is such that:

1) it is strictly plurisubharmonic (i.e. $2 i \partial \bar{\partial} \tau=d d^{c} \tau>0$ );

2) there exists a smooth function $f:(0, \infty) \rightarrow \mathbb{R}$ with $d f \neq 0$ such that the composition $u:=f \circ \tau$ is a plurisubharmonic solution to the Monge-Ampère equation $(\partial \bar{\partial} u)^{n}=0$. 
Furthermore if $M$ is of Type 1 , then $u$ has a logarithmic singularity along $S_{o}$ (i.e. $u:=\log \tau$ ), while when $M$ is of Type 2 or of Type 3, $u$ has a "square root" singularity along $S_{o}$ (i.e. $u:=\sqrt{\tau})$.

For the almost homogeneous manifolds of Type 1 or Type 2, the existence of such exhaustion is either well known or not surprising. What is new in this result are the examples given by the manifolds of Type 3 and the unified approach for the construction of the exhaustion, based on properties of group actions and on the detailed analysis of the complex structure of the Morimoto-Nagano manifolds due to Stenzel ([32]; see also [20]).

The main motivation for presenting in a unified way such three families of examples comes from the need of a common approach to the problem of deformation and of rigidity for complex manifolds with plurisubharmonic exhaustions that are solutions of the complex homogeneous Monge-Ampère equation. There are a number of elements that play a crucial role into the picture, among which we name:

a) the nature of the minimal set and of the singularity of the exhaustion along it;

b) the nature of the leaves of the foliation associated to the exhaustion (either parabolic or hyperbolic Riemann surfaces) which, in turn, depends on its upper boundedness or unboundedness.

In $\S 4$ we start such study of deformations, providing the set up and defining the main tools for such investigation, in particular the appropriate deformation tensors. Here, we suitably extend the notions introduced in [24] and based on the work of Bland and Duchamp [5], for the analysis of deformations of the so-called manifolds of circular type, an important family of complex manifolds that is included in the class of examples of Type 1. In Theorem 4.2 we give a first result which provides informations on the deformability of examples of Type 1, Type 2 and Type 3 according to the nature of the minimal set of the exhaustion.

\subsection{Notation.}

An $n$-dimensional complex manifold $M$ is considered as a pair $(M, J)$, where $J$ is the $(1,1)$ tensor field that gives the complex structure. The operator $d^{c}:=d_{J}^{c}$ is defined on $k$-forms by $d^{c}=J \circ d \circ J$, so that $d d^{c}=2 i \partial \bar{\partial}$.

A CR manifold of hypersurfaces type will be indicated as triple $(N, \mathcal{D}, J)$, given by a real manifold $N$ of odd dimension, a codimension one distribution $\mathcal{D} \subset T N$ and a smooth family $J$ of complex structures $J_{x}: \mathcal{D}_{x} \rightarrow \mathcal{D}_{x}, x \in N$, satisfying the integrability conditions $[J X, Y]+[X, J Y] \in \mathcal{D}$ and $[J X, J Y]-[X, Y]-J[J X, Y]-J[X, J Y]=0$ for any $X, Y \in$ $\mathcal{D}$. The holomorphic distribution of $(N, \mathcal{D}, J)$ is the subbundle $\mathcal{D}^{10} \subset T^{\mathbb{C}} N$ of the $+i$ eigenspaces of $\mathbb{C}$-linear maps $J_{x}: \mathcal{D}_{x}^{\mathbb{C}} \rightarrow \mathcal{D}_{x}^{\mathbb{C}}$. We recall that a $\mathrm{CR}$ manifold $(N, \mathcal{D}, J)$ is Levi non-degenerate if and only it the underlying real distribution $\mathcal{D}$ is contact.

A complex space $\mathcal{X}$ is actually a pair $\left(\mathcal{X}, \mathcal{O}_{\mathcal{X}}\right)$, where $\mathcal{X}$ is a Hausdorff topological space and $\pi: \mathcal{O}_{\mathcal{X}} \rightarrow \mathcal{X}$ is the sheaf of local $\mathbb{C}$-algebras, characterizing the complex space. Any complex manifold $M$ is identified with the complex space $\left(M, \mathcal{O}_{M}\right)$, with $\mathcal{O}_{M}$ sheaf of germs of local holomorphic functions of $M$. It is well known $([30,9,21])$ that if $\left(\mathcal{X}, \mathcal{O}_{\mathcal{X}}\right)$ is a complex space carrying a $\mathcal{C}^{\infty}$-exhaustion $\tau: \mathcal{X} \rightarrow[0, \infty)$, which is strictly plurisubharmonic outside a compact set, then it always admits a Remmert reduction, i.e. a pair $\left(\left(\mathcal{Y}, \mathcal{O}_{\mathcal{Y}}\right), \pi\right)$, formed by a Stein space $\left(\mathcal{Y}, \mathcal{O}_{\mathcal{Y}}\right)$ and a proper surjective holomorphic map $\pi: \mathcal{X} \rightarrow \mathcal{Y}$ such that: a) $\pi: \mathcal{X} \rightarrow \mathcal{Y}$ has connected fibers, b) $\pi_{*}\left(\mathcal{O}_{\mathcal{X}}\right)=\mathcal{O}_{\mathcal{Y}}$ and c) for any holomorphic map $f: \mathcal{X} \rightarrow \mathcal{Z}$ into a Stein space $\left(\mathcal{Z}, \mathcal{O}_{\mathcal{Z}}\right)$, there is a unique holomorphic map $f^{\prime}: \mathcal{Y} \rightarrow \mathcal{Z}$ such that $f=f^{\prime} \circ \pi$. Geometrically, the projection map $\pi: \mathcal{X} \rightarrow \mathcal{Y}$ collapses all positivedimensional compact analytic sets of $\mathcal{X}$. 


\section{Monge-Ampère SPACES}

In this paper, our interest is focused on the following class of complex manifolds with plurisubharmonic exhaustions.

Definition 2.1. Let $\widetilde{\mathcal{X}}$ be a complex manifold. A Monge-Ampère $\mathcal{C}^{\infty}$ exhaustion for $\tilde{\mathcal{X}}$ is a $\mathcal{C}^{\infty}$ exhaustion $\tau: \widetilde{\mathcal{X}} \rightarrow[0, T)$, possibly with $T=\infty$, whose restriction to $\widetilde{\mathcal{X}} \backslash\{\tau=0\}$ verifies:

1) it is strictly plurisubharmonic (i.e. $2 i \partial \bar{\partial} \tau=d d^{c} \tau>0$ )

2) there exists a smooth function $f:(0, \infty) \rightarrow \mathbb{R}$ with $d f \neq 0$ such that the composition $u:=f \circ \tau$ is a solution to the Monge-Ampère equation $(\partial \bar{\partial} u)^{n}=0$ and satisfies the non-negativity condition $2 i \partial \bar{\partial} u \geq 0$.

A Stein space $\mathcal{X}$ is a Monge-Ampère space if it is the space of a Remmert reduction $\pi$ : $\widetilde{\mathcal{X}} \rightarrow \mathcal{X}$ from a complex manifold $\widetilde{\mathcal{X}}$ with a Monge-Ampère $\mathcal{C}^{\infty}$ exhaustion $\tau: \widetilde{\mathcal{X}} \rightarrow[0, T)$. If $\mathcal{X}$ is a smooth complex manifold, we call it Monge-Ampère manifold.

If $\mathcal{X}$ is a Monge-Ampère space, the continuous function

$$
\tau^{\prime}: \mathcal{X} \rightarrow[0, T), \quad \tau^{\prime}(x):=\tau(y) \text { for some } y \in \pi^{-1}(x)
$$

is called Monge-Ampère $\mathcal{C}^{0}$ exhaustion of $\mathcal{X}$. The level set $\tau^{\prime-1}(0)=\pi\left(\tau^{-1}(0)\right)$ is the soul of $\mathcal{X}$ determined by $\tau$. By construction, the exhaustion $\tau^{\prime}$ is surely of class $\mathcal{C}^{\infty}$ on the complementary set of the soul.

A modeling example for the class of Monge-Ampère spaces is the complex Euclidean space $\mathbb{C}^{n}$, equipped with the standard exhaustion

$$
\tau_{o}: \mathbb{C}^{n} \rightarrow[0,+\infty), \quad \tau_{o}(z)=\|z\|^{2} .
$$

Indeed, $\mathbb{C}^{n}$ is the Remmert reduction of the blow up $\pi: \widetilde{\mathbb{C}}^{n} \rightarrow \mathbb{C}^{n}$ of $\mathbb{C}^{n}$ at the origin and the unique smooth function $\tau: \widetilde{\mathbb{C}} \rightarrow[0,+\infty)$, which extends the function $\left.\tau_{o}\right|_{\mathbb{C}^{n} \backslash\{0\}} \rightarrow(0,+\infty)$

at all points of $\pi^{-1}(0) \simeq \mathbb{C} P^{n-1}$, is a Monge-Ampère $\mathcal{C}^{\infty}$ exhaustion for $\widetilde{\mathbb{C}}^{n}$. The function $u=f \circ \tau$, which satisfies (2) in this case, is $u(x)=\log \left(\|x\|^{2}\right)$. The soul is the singleton $\{0\}$.

Other important examples of Monge-Ampère manifolds are the domains of circular type (22, 23, 24]), a class which naturally includes all circular domains and all strictly convex domains of $\mathbb{C}^{n}$. As for $\mathbb{C}^{n}$, each manifold of circular type is the Remmert reduction of its blow-up at a fixed point $x_{o}$, called center, and it has a Monge-Ampère $\mathcal{C}^{0}$-exhaustion, whose corresponding soul consists only of the center $x_{o}$.

Examples of Monge-Ampère manifolds with souls containing more than one point are given by the so-called Morimoto-Nagano spaces. They are the complex manifolds $(N, J)$, in which $N=T(G / K)$ is the tangent bundle of a CROSS $G / K$ and $J$ is the $G^{\mathbb{C}}$-invariant complex structure, determined by the natural identification of $T(G / K) \simeq$ $G^{\mathbb{C}} / K^{\mathbb{C}}$. A Morimoto-Nagano space $(N, J)$ is equipped with a Monge-Ampère exhaustion $\tau: T(G / K) \rightarrow[0,+\infty)$ for which $\tau^{-1}(0)$ coincides with the zero section of $N=T(G / K)$ and is therefore a totally real manifold of maximal dimension. In these examples, the exhaustion is actually $\mathcal{C}^{\infty}$ at all points and the manifold is the Remmert reduction of itself.

\section{Monge-Ampère exhaustions of Almost homogeneous MANifoldS}

\subsection{Almost homogeneous manifolds with cohomogeneity one actions.}

Let $M$ be an $n$-dimensional complex manifold and $G$ a real Lie group of biholomorphisms of $M$, which acts on $M$ of cohomogeneity one, that is with principal orbits that 
are real hypersurfaces of $M$. Since any principal orbit $G \cdot x$ is a real hypersurface of a complex manifold, it is naturally equipped with a $G$-invariant induced $\mathrm{CR}$ structure $(\mathcal{D}, J)$ of hypersurface type. If all principal $G$-orbits are strongly pseudoconvex, we say that the cohomogeneity one action is of strongly pseudoconvex type.

A notion that is strictly related with the cohomogeneity one actions is the following. Let $G^{\mathbb{C}}$ be a complex Lie group of biholomorphisms of $M$. The complex manifold is called almost homogeneous for to the $G^{\mathbb{C}}$-action (shortly, almost $G^{\mathbb{C}}$-homogeneous) if there is a $G^{\mathbb{C}}$-orbit which is open and dense in $M$.

It is clear that any homogeneous complex manifold $M=G^{\mathbb{C}} / H$ is almost homogeneous, but there are many examples of almost homogeneous manifolds that are not homogenous. Many such examples are indeed offered by cohomogeneity one actions. Assume that $G \subset G^{\mathbb{C}}$ is a compact real form of a reductive complex Lie group $G^{\mathbb{C}}$ and that there is a cohomogeneity one $G$-action on a compact complex manifold $M$. If $x \in M$ is a regular point for the $G$-action, the $G^{\mathbb{C}}$-orbit $G^{\mathbb{C}} \cdot x$ is a complex submanifold of $M$ containing the real hypersurface $G \cdot x$. It is therefore open in $M$. As a consequence of standard facts on the orbit space of cohomogeneity one actions (see e.g. [7, 28, 29]), one can see that such open orbit $G^{\mathbb{C}} \cdot x$ is dense but, in general, not equal to $M$. More precisely, $G^{\mathbb{C}} \cdot x=M$ if and only if the real Lie group $G$ has no complex singular orbits in $M$.

\subsection{Three important classes of almost homogeneous manifolds.}

We now focus on a special class of compact almost homogeneous $G^{\mathbb{C}}$-manifolds with a compact real form $G \subset G^{\mathbb{C}}$ acting of cohomogeneity one.

Let $M$ be a compact complex manifold with a cohomogeneity one holomorphic $G$-action of strongly pseudoconvex type. Each principal $G$-orbit $N=G \cdot x \subset M$ has the following two important properties:

- it is a compact homogeneous $G$-manifold, identifiable with a coset space $N=G / H$;

- it has an induced $G$-invariant strongly pseudoconvex CR structure $(\mathcal{D}, J)$.

The classification (up to coverings) of compact homogeneous CR manifolds $(G / H, \mathcal{D}, J)$ with these two properties has been determined in [2, 3]. From this classification and other important properties of almost homogeneous manifolds, proved in [14, 15, 1], in principle one can get a complete description of all compact almost $G^{\mathbb{C}}$-homogeneous manifolds $M$ with cohomogeneity one $G$-actions of strongly pseudoconvex type. Such description is given explicitly in [29] under the assumption that the first Betti number is $b_{1}(M)=0$. As it is pointed out in [1], the cases with $b_{1}(M)=p \neq 0$ are fibered bundle over $p$-dimensional complex tori, with a fibre $M^{\prime}$ which is an almost homogeneous manifold with $b_{1}\left(M^{\prime}\right)=0$.

According to [29], any compact almost homogeneous manifold $M$ with strongly pseudoconvex, cohomogeneity one $G$-actions and with $b_{1}(M)=0$ belongs to one of the following disjoint three classes. Here, the complex structure $J$ of $M$ is the natural $G$-invariant complex structure.

3.2.1. Almost homogeneous manifolds with two ends. Consider the $\mathbb{C} P^{1}$-bundles of the form

$$
\pi: M=G^{\mathbb{C}} \times_{P, \rho} \mathbb{C} P^{1} \longrightarrow G^{\mathbb{C}} / P,
$$

where:

a) $G^{\mathbb{C}} / P$ is a flag manifold (i.e. a homogeneous quotient of a complex semisimple Lie group $G^{\mathbb{C}}$ by a parabolic subgroup $P \subset G^{\mathbb{C}}$ ), equipped with a compact real form $G \subset G^{\mathbb{C}}$ and a fixed choice of a $G$-invariant Kähler metric $g$ on $G^{\mathbb{C}} / P$; 
b) $\rho: P \rightarrow$ Aut $\left(\mathbb{C} P^{1}\right)$ is a biholomorphic action on $\mathbb{C} P^{1}$ of the isotropy group $P$, such that $\left.\rho\right|_{G \cap P}: G \cap P \rightarrow$ Aut $\left(\mathbb{C} P^{1}\right)$ coincides with the standard cohomogeneity one action of $T^{1}=\rho(G \cap P)$ on $\mathbb{C} P^{1}$.

For each such $\mathbb{C} P^{1}$-bundle, the compact real form $G \subset G^{\mathbb{C}}$ acts transitively on the flag manifold $F=G^{\mathbb{C}} / P$ and of cohomogeneity one on $M$. There are exactly two singular $G$ orbits, say $S, S^{\prime}$, both of them complex and $G$-equivalent to the flag manifold $F=G^{\mathbb{C}} / P=$ $G / G \cap P$. Their intersections with a fibre of $\pi: M=G^{\mathbb{C}} \times_{P, \rho} \mathbb{C} P^{1} \longrightarrow G^{\mathbb{C}} / P$ are the two singular orbits of the standard action of $T^{1}$ on $\mathbb{C} P^{1}$. The action of $G^{\mathbb{C}}$ has three disjoint orbits: $M_{\text {reg }}:=M \backslash\left(S \cup S^{\prime}\right.$ ) (which is open and dense), $S$ and $S^{\prime}$.

A manifold of this kind is usually called with two ends, since any singular $G$-orbit that is complex is referred as an end of the manifold. It is known that any other almost homogeneous manifold, which satisfies the above conditions and for which there are two complex singular $G$-orbits, admits a blow up, which is $G$-equivalent to $\mathbb{C} P^{1}$-bundles described above (29], Thm. 2.4). We shortly call such manifolds almost homogeneous manifolds with two ends and those as in (3.1) in canonical form.

The simplest example in this class is the blow up $\widetilde{\mathbb{C} P^{n}}$ of $\mathbb{C} P^{n}$ at a point $\left[x_{o}\right]$. Indeed, it is an almost homogeneous manifold with two ends with

$$
\begin{aligned}
& G=\mathrm{SU}_{n}, \quad G^{\mathbb{C}}=\mathrm{SL}_{2}(\mathbb{C}), \\
& G^{\mathbb{C}} / P:=\mathrm{SL}_{n}(\mathbb{C}) / P \simeq \mathbb{C} P^{n-1}, \quad \text { where } P:=\left\{A \in \mathrm{SL}_{n}(\mathbb{C}): A \cdot x_{o}=x_{o}\right\}
\end{aligned}
$$

The singular $G$-orbits are both biholomorphic to $\mathbb{C} P^{n-1}$, one given by the exceptional divisor at $\left[x_{o}\right]$, the other by the hyperplane $\pi_{o}=\left\{[x]: x \in\left(x_{o}\right)^{\perp}\right\}$.

3.2.2. Compactifications of Morimoto-Nagano spaces. It is the class of compact complex manifolds, given by the infinite sequences of manifolds

$$
\mathbb{C} P^{n}, \quad \mathcal{Q}^{n}=\left\{[z] \in \mathbb{C} P^{n+1}:{ }^{t} z z=0\right\}, \quad \mathbb{C} P^{n} \times \mathbb{C} P^{n}, \operatorname{Gr}_{2,2 n}(\mathbb{C}),
$$

together with the Cayley projective plane

$$
E I I I=E_{6} / \mathrm{SO}_{2} \cdot \mathrm{Spin}_{10} .
$$

Each of these manifold is a $G$-invariant compactification of a Morimoto-Nagano space for an appropriate compact simple Lie group $G$. More precisely,

a) $\mathbb{C} P^{n}$ is the $\mathrm{SO}_{n}$-invariant complex compactification of $T \mathbb{R} P^{n}$,

b) $\mathcal{Q}^{n}$ is the $\mathrm{SO}_{n}$-invariant complex compactification of $T S^{n}$,

c) $\mathbb{C} P^{n} \times \mathbb{C} P^{n}$ is the $\mathrm{SO}_{n}$-invariant complex compactification of $T \mathbb{C} P^{n}$,

d) $\mathrm{Gr}_{2,2 n}(\mathbb{C})$ is the $\mathrm{Sp}_{n}$-invariant compactifications of $T \mathbb{H} P^{n}$,

e) $E I I I$ is the $\mathrm{F}_{4}$-invariant complex compactification of $T \mathbb{O} P^{2}$.

In all these cases, $G$ acts of cohomogeneity one of strongly pseudoconvex type. There are two singular $G$-orbits, one complex, the other totally real. The former is the complex manifold which is complementary to the tangent bundle TS of the CROSS $S=G / K$, the latter is the zero section of $T S$ and is therefore identifiable with $S=G / K$. Since only one singular $G$-orbit is complex, these manifolds are said to be with one end.

We remark that, by the results of Morimoto and Nagano ([19]), $\mathbb{C}^{n}$, the unit ball $\mathbb{B}^{n} \subset$ $\mathbb{C}^{n}$ and the Morimoto-Nagano spaces are the only Stein manifolds on which there is a biholomorphic cohomogeneity one action of strongly pseudoconvex type for a compact Lie group $G$. This is one of the main reasons of interest for this class. 
3.2.3. Almost homogeneous manifolds with one end of mixed type. This class consists of the almost homogeneous manifolds constructed as follows. Let $G$ be a compact Lie group and $\widehat{M}$ a homogeneous $G$-bundle of the form

$$
\pi: \widehat{M}=G \times_{G_{Q}, \rho} F \longrightarrow G / G_{Q},
$$

where the basis $G / G_{Q}$, the fibre $F$ and the representation $\rho: G_{Q} \rightarrow$ Aut $(F)$ form one of the triples listed in Table 1 . There, the map $\rho$ is indicated only by the group $\rho\left(G_{Q}\right)$, which in all cases has to be considered as a group of projective transformations of a projective space $\mathbb{C} P^{s}$ or $\mathbb{C} P^{s+1}$, depending on whether $F=\mathbb{C} P^{s}$ or $F=\mathcal{Q}^{s} \subset \mathbb{C} P^{s+1}$.

The almost homogeneous manifolds of this class are those having the form (3.2) together with all other complex $G$-manifolds with exactly one complex singular $G$-orbit and admitting a manifold $\widehat{M}$ as a blow-up along such $G$-orbit. By a direct inspection of the Levi forms of the regular $G$-orbits (they can be determined from the explicit descriptions in [4]), one can check that all of them are of strongly pseudoconvex type. Each manifold of this third class has two singular $G$-orbits $S, S^{\prime}$, the first complex, the second neither complex nor totally real. However, the intersection of $S^{\prime}$ with each fibre $F_{x}=\pi^{-1}(x)$ (which is an almost homogeneous space of the second class) is a totally real submanifold $G$-equivalent to $\mathbb{R} P^{n}$ or $S^{n}$. Due to this, the manifolds of this third class are called with one end and of mixed type and those as in (3.2) in canonical form.

\begin{tabular}{|c|c|c|c|}
\hline & $G / G_{Q}$ & $F$ & $\rho(Q)$ \\
\hline \hline$I_{1}$ & $\mathrm{SU}_{n} / \mathrm{S}\left(\mathrm{U}_{2} \times \mathrm{U}_{n-2}\right)$ & $\mathbb{C} P^{2}$ & $\mathrm{SO}_{3}$ \\
\hline$I_{2}$ & $\mathrm{SU}_{n} / \mathrm{S}\left(\mathrm{U}_{2} \times \mathrm{U}_{n-2}\right)$ & $\mathcal{Q}^{2}$ & $\mathrm{SO}_{3}$ \\
\hline$I I$ & $\begin{array}{r}\left.\left(\mathrm{SU}_{p} / \mathrm{S}_{2} \times \mathrm{U}_{p-2}\right)\right) \times\left(\mathrm{SU}_{q} / \mathrm{S}\left(\mathrm{U}_{2} \times \mathrm{U}_{q-2}\right)\right) \\
p+q>4\end{array}$ & $\mathbb{C} P^{3}$ & $\mathrm{SO}_{4} / \mathbb{Z}_{2}$ \\
\hline$I I I$ & $\left.\mathrm{SU}_{n} / \mathrm{S}_{4} \times \mathrm{U}_{n-4}\right) \quad n>4$ & $\mathbb{C} P^{5}$ & $\mathrm{SO}_{6} / \mathbb{Z}_{2}$ \\
\hline$I V_{1}$ & $\mathrm{SO}_{10} / \mathrm{SO}_{2} \times \mathrm{SO}_{8}$ & $\mathbb{C} P^{7}$ & $\mathrm{SO}_{8} / \mathbb{Z}_{2}$ \\
\hline$I V_{2}$ & $\mathrm{SO}_{10} / \mathrm{SO}_{2} \times \mathrm{SO}_{8}$ & $\mathcal{Q}^{7}$ & $\mathrm{SO}_{8}$ \\
\hline$V_{1}$ & $\mathrm{E}_{6} / \mathrm{SO}_{2} \times \mathrm{Spin}_{10}$ & $\mathbb{C} P^{9}$ & $\mathrm{SO}_{10} / \mathbb{Z}_{2}$ \\
\hline$V_{2}$ & $\mathrm{E}_{6} / \mathrm{SO}_{2} \times \mathrm{Spin}_{10}$ & $\mathcal{Q}^{9}$ & $\mathrm{SO}_{10}$ \\
\hline
\end{tabular}

Table 1

Remark 3.1. From Table 1, cases $I I$ and $I I I$ are the only ones with no counterparts with quadrics as fibers. The reason becomes manifest if one recalls how Table 1 derives from the previous results on almost homogeneous manifolds and homogeneous CR structures.

By [15, if a compact almost homogeneous $G^{\mathbb{C}}$-manifold $M$ has a cohomogeneity one $G$-action and just one end, then it is either a compactification of a Morimoto-Nagano space or a fiber bundle over a flag manifold $G / G_{Q}$. In this second case, the fiber $F$ is either a compactification of a Morimoto-Nagano space or admits a cohomogeneity one $G$-action with a single isolated fixed point. Since each regular $G$-orbits in $M$ are homogeneous compact CR manifolds, up to a covering, all of them are further constrained to be $G$-equivariantly equivalent to a Levi non-degenerate homogeneous CR manifolds of the classification in [2].

This last fact gives a lot of restrictions on the group $G$ and the fiber $F$. In fact, one gets that there are very few possibilities for the triples $\left(G / G_{Q}, F, \rho\left(G_{Q}\right)\right)$, in which $F$ must be either $\mathbb{C} P^{s}$ or $\mathcal{Q}^{s} \subset \mathbb{C} P^{s+1}$ with $s=2,3,5,7$ or 9 , the group $\rho\left(G_{Q}\right)$ either $\mathrm{SO}_{s} / \mathbb{Z}$ or $\mathrm{SO}_{s}$ and $G / G_{Q}$ must be one of the five possibilities appearing in Table I, one per each of the five possible cases for the dimension $s$ of $F$. An additional restriction comes from the fact 
that $G_{Q}$ must admit a representation $\rho: G_{Q} \rightarrow$ Aut $(F)$ with $\rho\left(G_{Q}\right)=G_{Q} /$ ker $\rho$ equal to $\mathrm{SO}_{s} / \mathbb{Z}_{2}$ or $\mathrm{SO}_{s}$. For all five cases for $s$, there exists a closed normal subgroup $N \subset G_{Q}$ so that $G_{Q} / N=\mathrm{SO}_{s} / \mathbb{Z}_{2}$, but only for three of them there exists a normal subgroup $N$ so that $G_{Q} / N=\mathrm{SO}_{s}$. Cases $I I$ and $I I I$ are those where there is no such normal subgroup.

\subsection{A new class of Monge-Ampère spaces.}

From now on, we restrict to the complex manifolds described in 33.2 .1 , 3.2.2 and 3.2 .3 . For each of them we refer to $G$ as the group of the cohomogeneity one action.

As we already mentioned, on each such manifold at least one of the two singular $G$-orbits is complex. Let $S \subset M$ be such orbit and denote by $M_{O}:=M \backslash S$ its complementary set. By definition, it is a complex manifold, on which

- $G$ has a cohomogeneity one action of strongly pseudoconvex type;

- $G^{\mathbb{C}}$ acts either transitively on $M_{o}$ (this occurs when the second singular $G$-orbit $S^{\prime} \subset M$ is not complex) or with an open and dense orbit.

In addition to this, the following crucial property holds.

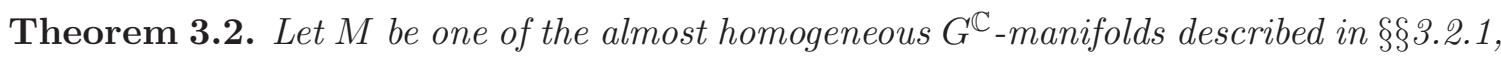
3.2.2, 3.2.3 in canonical form and $S=G \cdot x \subset M$ a complex singular $G$-orbits. Then:

i) The complementary set $M_{o}=M \backslash S$ admits a G-invariant Monge-Ampère exhaustion $\tau: M_{o} \rightarrow[0, \infty)$;

ii) This exhaustion is the unique (up to a scaling factor) G-invariant Monge-Ampère exhaustion $\tau$ satisfying the following three conditions:

$\alpha$ ) it satisfies (2) of Definition 2.1 with $f(t)=\log (t)$ in case both singular $G$-orbits of $M=M_{o} \cup S$ are complex and $f(t)=\sqrt{t}$ otherwise;

$\beta)$ the level set $\{\tau=0\}$ coincides with the second singular $G$-orbit $S^{\prime}$ of $M$;

$\gamma$ ) if $S^{\prime}$ is not complex, then $u=f \circ \tau=\sqrt{\tau}$ admits a continuous extension at each point $x_{o} \in\{\tau=0\}$; if $S^{\prime}$ is complex, then for each $x_{o} \in\{\tau=0\}$, there is a system of complex coordinates $z=\left(z^{i}\right)$ centered at $x_{o}$, in which $u=f \circ \tau=\log \tau$ has a logarithmic singularity at $x_{o}$, i.e.

$$
u(z)=\log \tau(z)=\log \|z\|+O(1) .
$$

Thus, any Remmert reduction $\mathcal{M}_{o}$ of one such manifold $M_{o}$ is a Monge-Ampère space.

We remark that this theorem gives a whole new class of examples of Monge-Ampère spaces $(\mathcal{X}, \tau)$. Indeed, all known examples of Monge-Ampère spaces are complex $n$ dimensional manifolds with a soul $\mathcal{S}$, which is either formed by an isolated point (it is what occurs in a manifold of circular type) or a totally real submanifold of maximal dimension, i.e. with $\operatorname{dim}_{\mathbb{R}} \mathcal{S}=\frac{1}{2} \operatorname{dim}_{\mathbb{R}} \mathcal{X}$ (it is the case of the Morimoto-Nagano spaces). But, by the above theorem, we see that each manifold $M_{o}=M \backslash S$ determined by a manifold $M$ in canonical form of the third class has a Remmert reduction which is a Monge-Ampère space with a soul $\mathcal{S}$ that is neither a point nor a totally real submanifold of maximal dimension.

The proof of Theorem 3.2 is based on some properties of almost homogeneous spaces, which we recall in the next subsections. We begin by introducing some additional notation.

3.3.1. Notational issues. Let $G^{\mathbb{C}}$ be the complexification of the semisimple Lie group $G$ and $\mathfrak{g}=\operatorname{Lie}(G), \mathfrak{g}^{\mathbb{C}}=\mathfrak{g}+i \mathfrak{g}=\operatorname{Lie}\left(G^{\mathbb{C}}\right)$. We denote by $\mathcal{B}$ the Cartan-Killing form of $\mathfrak{g}^{\mathbb{C}}$ and for any subspace $\mathfrak{v} \subset \mathfrak{g}^{\mathbb{C}}$, we indicate by $\mathfrak{v}^{\perp}$ its $\mathcal{B}$-orthogonal complement in $\mathfrak{g}^{\mathbb{C}}$. The same notation is used for the $\mathcal{B}$-orthogonal complements of subspaces of $\mathfrak{g}$. 
For each $X \in \mathfrak{g}^{\mathbb{C}}=\mathfrak{g}+i \mathfrak{g} \subset \operatorname{Lie}\left(\right.$ Aut $\left(M_{o}\right)$ ), we denote by $\widehat{X} \in \mathfrak{X}\left(M_{o}\right)$ the corresponding infinitesimal transformation of $M_{o}$, i.e. the unique complete vector field whose flow $\Phi_{t}^{\widehat{X}}$, $t \in \mathbb{R}$, is the family of diffeomorphisms

$$
\Phi_{t}^{\widehat{X}}: M_{o} \rightarrow M_{o}, \quad \Phi_{t}^{\widehat{X}}(x):=\exp (t X) \cdot x .
$$

We recall that the map ${ }^{\widehat{ }}: \mathfrak{g}^{\mathbb{C}} \longrightarrow \mathfrak{X}\left(M_{o}\right)$ between $\mathfrak{g}^{\mathbb{C}}$ and the space of vector fields of $M_{o}$ is an injective anti-homomorphism of Lie algebras, i.e. $[\widehat{X}, \widehat{Y}]=-\widehat{[X, Y]}$ for all $X, Y \in \mathfrak{g}^{\mathbb{C}}$.

Consider now a regular point $x_{o} \in M_{o}$ and identify $G \cdot x_{o}$ with the coset space $G \cdot x_{o}=G / L$ with $x_{o} \simeq e \cdot L$. We recall that the surjective linear map

$$
\imath: \mathfrak{g} \longrightarrow T_{x_{o}}\left(G \cdot x_{o}\right)=T_{e L} G / L, \quad \imath(X)=\left.\widehat{X}\right|_{x_{o}}
$$

induces an isomorphism between the vector space $\mathfrak{l}^{\perp} \subset \mathfrak{g}$, complementary to $\mathfrak{l}$, and the tangent bundle of $G \cdot x_{o}$ at $x_{o}$. In the following, we constantly use such isomorphism to identify these vector spaces. In this way, the subspace $\mathcal{D}_{x_{o}} \subset T_{x_{o}}\left(G \cdot x_{o}\right)$ and the complex structure $J_{x_{o}}: \mathcal{D}_{x_{o}} \rightarrow \mathcal{D}_{x_{o}}$ of the $\mathrm{CR}$ structure $(\mathcal{D}, J)$ are identified with

- an $\operatorname{Ad}_{L^{-}}$-invariant codimension one real subspace $\mathfrak{m} \subset \mathfrak{l}^{\perp}$;

- an $\operatorname{Ad}_{L}$-invariant complex structure $J: \mathfrak{m} \rightarrow \mathfrak{m}$.

Choosing a unitary vector $Z \in \mathfrak{l}^{\perp} \cap \mathfrak{m}^{\perp}$ (it is $\operatorname{Ad}_{L^{-}}$invariant and unique up to a sign), we get an $\mathrm{Ad}_{L}$-invariant decomposition

$$
\mathfrak{g}=\mathfrak{l}+\mathfrak{l}^{\perp}=\mathfrak{l}+(\mathfrak{m}+\mathbb{R} Z) .
$$

Using the fact that $\mathcal{D}$ is contact, one can show that $\mathfrak{l}+\mathbb{R} Z=C_{\mathfrak{g}}(Z)$ (see e.g. 4], §3.1).

3.3.2. Distinguished curves in the above three classes of almost homogeneous manifolds. Consider now the infinitesimal transformation $J \widehat{Z}=\widehat{i Z}$ corresponding to $i Z \in \mathfrak{g}^{\mathbb{C}}$ and let

$$
\eta: \mathbb{R} \longrightarrow M_{o}, \quad \eta_{t}:=\exp (i t Z) \cdot x_{o}=\Phi_{t}^{J \widehat{Z}}\left(x_{o}\right) .
$$

By Thms. 3.4 and 3.7 in [31, the curve $\eta$ has the following crucial properties.

(1) It intersects each regular $G$-orbit of $M_{o}=M \backslash S$; in fact, there exists a $G$-invariant Kähler metric $g$ on $M$, with the property that $\eta_{t}$ is a reparameterization of a geodesic of $g$ orthogonal to all regular $G$-orbits.

(2) If $S \subset M$ is the only complex singular $G$-orbit, then $\eta$ intersects the non-complex singular $G$-orbit $S^{\prime} \subset M_{o}$; in this case, there is no loss of generality if we change the starting point of $\eta$ and assume that $x_{o}=\eta_{0}$ is in $S^{\prime}$.

(3) If both singular $G$-orbits $S, S^{\prime}$ are complex, then $\eta$ intersects neither of them; however $\lim _{t \rightarrow+\infty} \eta_{t}$ is either in $S$ or $S^{\prime}$; changing $Z$ into $-Z$, we may always assume that $\lim _{t \rightarrow+\infty} \eta_{t} \in S^{\prime}$.

(4) Each element in the isotropy $G_{\eta_{t}}$ of a regular point $\eta_{t}$ fixes all other points of the curve; this implies that the space $\mathfrak{I}^{\perp} \simeq T_{\eta_{t}} G \cdot \eta_{t}$ is the same for all $G$-regular points of the curve $\eta$; there is also a canonical isomorphism $\mathcal{D}_{\eta_{t}}$ between the spaces $\mathcal{D}_{\eta_{t}}$ of the CR distributions of the $G$-orbit $G \cdot \eta_{t}$, so that they are all identifiable with a fixed vector subspace $\mathfrak{m} \subset \mathfrak{l}^{\perp}$, independent on $t$.

(5) If $M=M_{o} \cup S$ is an almost homogeneous manifold with one end and of mixed type, then $\eta_{t}$ is entirely included in a single fiber of the projection $\pi: M \rightarrow G / G_{Q}$ over the flag manifold $G / G_{Q}$ described in Table 1 . 
3.3.3. The distinguished curves of Morimoto-Nagano spaces. Assume now that $M=M_{o} \cup S$ is the compactification of a Morimoto-Nagano space, so that $M_{o}=T S^{\prime}$ for a CROSS $S^{\prime}=G / K$. Let $\mathfrak{k}=\operatorname{Lie}(K)$ and $\mathfrak{g}=\mathfrak{k}+\mathfrak{p}$ the corresponding $\mathcal{B}$-orthogonal decomposition of $\mathfrak{g}$. We recall that $M_{o}=T S^{\prime}$ is $G$-equivariantly identifiable with $T S^{\prime} \simeq G \times_{K, \rho} \mathfrak{p}$. with $\rho(K)=\left.\operatorname{Ad}_{K}\right|_{\mathfrak{p}}$, and that the $G$-invariant complex structure of $T S^{\prime}$ is the pull-back $J=$ $\varphi^{*}\left(J_{o}\right)$ of the complex structure $J_{o}$ of $G^{\mathbb{C}} / K^{\mathbb{C}}$ by means of the $G$-equivariant diffeomorphism

$$
\varphi: T S^{\prime}=G \times_{K, \rho} \mathfrak{p} \longrightarrow G^{\mathbb{C}} / K^{\mathbb{C}}, \quad \varphi\left([(g, X)]_{K}\right):=\exp (i X) \cdot g K^{\mathbb{C}} .
$$

An explicit expression for $J$ has been determined by Stenzel in [32] (see also [20]) and it can be described as follow. Let

$$
\pi: G \times \mathfrak{p} \rightarrow G \times \times_{K, \rho} \mathfrak{p}
$$

be the natural quotient map. Then, for any $(g, X) \in G \times \mathfrak{p}$, the vectors $v \in T_{(g, X)}(G \times \mathfrak{p})$ can be described as pairs $v=\left(\left.Y\right|_{g}, V\right)$, with $Y \in \mathfrak{g}$ and $V \in \mathfrak{p}$. Consequently, for each $w \in T_{[(g, X)]_{K}}\left(G \times{ }_{K} \mathfrak{p}\right)$, there is a (non-unique) element $\left(\left.Y^{(w)}\right|_{g}, V^{(w)}\right) \in T_{(g, \mathfrak{p})}(G \times \mathfrak{p})$ with

$$
\pi_{*}\left(\left.Y^{(w)}\right|_{g}, V^{(w)}\right)=w .
$$

By [32], the tensor $J_{[(g, X)]_{K}}$ is the unique endomorphism of $T_{[(g, X)]_{K}} G \times_{K, \rho} \mathfrak{p}$ such that

$$
\begin{aligned}
& J(w)=\pi_{*}\left(-\left.\left(\mathcal{T}_{X}\right)^{-1}\left(V^{(w)}\right)\right|_{g}+\left.\mathcal{T}_{X}\left(\operatorname{ad}_{X}\left(Y^{(w) \mathfrak{k}}\right)\right)\right|_{g}, \mathcal{T}_{X}\left(Y^{(w) \mathfrak{p}}\right)\right) . \\
& \text { with } \mathcal{T}_{X}:=\left(\frac{\sin \operatorname{ad}_{X}}{\operatorname{ad}_{X}}\right)^{-1} \circ \cos \operatorname{ad}_{X},
\end{aligned}
$$

where for each $E \in \mathfrak{g}=\mathfrak{k}+\mathfrak{p}$, we denote by $E^{\mathfrak{k}}, E^{\mathfrak{p}}$ the $\mathcal{B}$-orthogonal projections into $\mathfrak{k}$ and $\mathfrak{p}$, respectively, and the notation $\sin \operatorname{ad}_{X}, \cos \operatorname{ad}_{X}$, etc. stand for the operators defined by power series. Note that for each $X$ in $\mathfrak{p}$ or in $\mathfrak{k}$, the linear operators

$$
\frac{\sin \operatorname{ad}_{X}}{\operatorname{ad}_{X}}: \mathfrak{g} \longrightarrow \mathfrak{g} \quad \text { and } \quad \cos \operatorname{ad}_{X}: \mathfrak{g} \longrightarrow \mathfrak{g}
$$

are invertible, even and preserve $\mathfrak{k}, \mathfrak{p}$. So, also $\mathcal{T}_{X}$ is invertible, even and preserves $\mathfrak{k}$ and $\mathfrak{p}$.

With the help of this information we may now give an explicit description for the curve $\eta: \mathbb{R} \rightarrow T S^{\prime}$, described in 3.3.2. For simplicity, assume that the identification $T S^{\prime} \simeq$ $G \times K, \rho \mathfrak{p}$ is done in such a way that the $G$-regular point $y_{o}=\eta_{1} \in M_{o} \backslash S^{\prime}$ has the form

$$
y_{o}=\left[\left(e, X_{o}\right)\right]_{K} \quad \text { for some } \quad 0 \neq X_{o} \in \mathfrak{p} .
$$

Then, the isotropy subalgebra $\mathfrak{l}:=\mathfrak{g}_{y_{o}}$ of $G$ at $y_{o}$ is $\mathfrak{l}=\mathfrak{k} \cap \operatorname{ker}\left(\operatorname{ad}_{X_{o}}\right)$. Moreover, since $S^{\prime}=G / K$ has rank one, we know that $\operatorname{ker}\left(\operatorname{ad}_{X_{o}}\right) \cap \mathfrak{p}=\mathbb{R} X_{o}$ so that the linear map

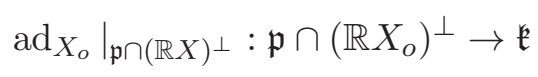

has trivial kernel. Consider now the vector subspaces $\mathfrak{p}_{1}, \mathfrak{p}_{2}, \mathfrak{m}^{\prime}$ of $\mathfrak{g}=\mathfrak{k}+\mathfrak{p}$ defined by

$$
\mathfrak{p}_{1}:=\mathfrak{p} \cap\left(\mathbb{R} X_{o}\right)^{\perp} \subset \mathfrak{p}, \quad \mathfrak{p}_{2}:=\operatorname{ad}_{X_{o}}\left(\mathfrak{p}_{1}\right) \subset \mathfrak{k}, \quad \mathfrak{m}^{\prime}:=\mathfrak{p}_{1}+\mathfrak{p}_{2} .
$$

Lemma 3.3. The subspace $\mathfrak{m}^{\prime}$ coincides with the $\operatorname{ad}_{X_{o}}$-invariant orthogonal complement $\mathfrak{m}^{\prime}=\left(\mathfrak{l}+\mathbb{R} X_{o}\right)^{\perp}$. This also implies that $\operatorname{ad}_{X_{o}}\left(\mathfrak{p}_{2}\right)=\mathfrak{p}_{1}$.

Proof. Since $\left[\mathfrak{l}, X_{o}\right]=0$ we have that $\mathcal{B}\left(\mathfrak{l}, \mathfrak{p}_{2}\right)=\mathcal{B}\left(\mathfrak{l},\left[X, \mathfrak{p}_{1}\right]\right)=-\mathcal{B}\left([\mathfrak{l}, X], \mathfrak{p}_{1}\right)=0$, i.e. $\mathfrak{p}_{2} \subset \mathfrak{l}^{\perp}$. This, together with the fact that $\mathfrak{p}_{2} \subset \mathfrak{k} \subset\left(\mathbb{R} X_{o}\right)^{\perp}$, implies that $\mathfrak{m}^{\prime}=\mathfrak{p}_{1}+\mathfrak{p}_{2} \subset$ $\left(\mathfrak{l}+\mathbb{R} X_{o}\right)^{\perp}$. The equality follow by counting dimensions. 
By this lemma, we have $\mathfrak{l}^{\perp}=\mathbb{R} X_{o}+\mathfrak{m}^{\prime}$. On the other hand, for each $Y_{1} \in \mathfrak{p}_{1}, Y_{2} \in \mathfrak{p}_{2}$,

$$
\begin{aligned}
\left(\widehat{Y_{1}+Y_{2}}\right)_{\left[\left(e, X_{o}\right)\right]_{K}} & =\left.\frac{d}{d t} \exp \left(t\left(Y_{1}+Y_{2}\right)\right) \cdot\left[\left(e, X_{o}\right)\right]_{K}\right|_{t=0}= \\
& =\pi_{*}\left(\left.\frac{d}{d t}\left(\exp \left(t\left(Y_{1}+Y_{2}\right)\right), X_{o}\right)\right|_{t=0}\right)=\pi_{*}\left(\left.Y_{1}\right|_{e}+\left.Y_{2}\right|_{e}, 0\right) .
\end{aligned}
$$

Hence, by (3.5)

$$
J\left({\widehat{Y_{1}+Y_{2}}}_{2}\right)_{\left[\left(e, X_{o}\right)\right]_{K}}=\pi_{*}\left(\left.\mathcal{T}_{X_{o}}\left(\operatorname{ad}_{X_{o}}\left(Y_{2}\right)\right)\right|_{e}, \mathcal{T}_{X_{o}}\left(Y_{1}\right)\right)
$$

We now observe that, since $\mathcal{T}_{X_{o}}$ is a series of even powers of $\operatorname{ad}_{X_{o}}$, by Lemma 3.3, it preserves $\mathfrak{p}_{1}$ and $\mathfrak{p}_{2}$. Hence, both $Y_{1}^{\prime}:=\mathcal{T}_{X_{o}}\left(\operatorname{ad}_{X}\left(Y_{2}\right)\right)$ and $Y_{2}^{\prime}:=\mathcal{T}_{X_{o}}\left(Y_{1}\right)$ are in $\mathfrak{p}_{1}$. Moreover, since $\left.\operatorname{ad}_{X_{o}}\right|_{\mathfrak{p}_{2}}: \mathfrak{p}_{2} \rightarrow \mathfrak{p}_{1}$ is a linear isomorphism, there exists $Y_{2}^{\prime \prime} \in \mathfrak{p}_{2} \subset \mathfrak{k}$ such that $Y_{1}^{\prime}=-\left[Y_{2}^{\prime \prime}, X_{o}\right]$. It follows that the vector (3.6) can be written as

$$
\begin{aligned}
J\left(\widehat{Y_{1}+Y_{2}}\right)_{\left[\left(e, X_{o}\right)\right]_{K}} & =\pi_{*}\left(\left.Y^{\prime}\right|_{e},-\left[Y_{2}^{\prime \prime}, X_{o}\right]\right)=\pi_{*}\left(\left.Y_{1}^{\prime}\right|_{e}+\left.Y_{2}^{\prime \prime}\right|_{e}, X_{o}\right)= \\
& =\left(\widehat{Y_{1}^{\prime}+Y_{2}^{\prime \prime}}\right)_{\left[\left(e, X_{o}\right)\right]_{K}} .
\end{aligned}
$$

This implies that under the natural isomorphism

$$
\imath: \mathbb{R} X_{o}+\mathfrak{m}^{\prime} \longrightarrow T_{y_{o}}\left(T S^{\prime}\right),
$$

the complex structure of the CR structure $(\mathcal{D}, J)$ of $T G \cdot y_{o}$ preserves the subspace $\mathfrak{m}^{\prime}$. By counting dimensions it follows that $\mathfrak{m}^{\prime}$ coincides with the $J$-invariant subspace $\mathfrak{m}$ of $\mathfrak{l}^{\perp}$ and that $\frac{X_{o}}{\mathcal{B}\left(X_{o}, X_{o}\right)}= \pm Z$. From this and (3.5), we also get that $\left.J \widehat{Z}\right|_{\left[\left(e, X_{o}\right)\right]_{K}}=\pi_{*}\left(0, \frac{ \pm X_{o}}{\mathcal{B}\left(X_{o}, X_{o}\right)}\right)$. Since the integral curve $\eta_{t}$ of $J \widehat{Z}$ satisfies the conditions $\eta_{0}=[(e, 0)]_{K}$ and $\eta_{1}=\left[\left(e, X_{o}\right)\right]_{K}$, we get that

$$
X_{o}=Z,\left.\quad J \widehat{Z}\right|_{\left[\left(e, X_{o}\right)\right]_{K}}=\pi_{*}(0, Z), \quad \eta_{t}=[(e, t Z)]_{K} .
$$

3.3.4. Proof of Theorem 3.2. We have now all ingredients for the proof. Consider the function $\tau: M_{o} \backslash S^{\prime} \longrightarrow \mathbb{R}$ defined as follows. Let $\eta: \mathbb{R} \rightarrow M_{o}$ be one of the curves defined in $\$ 3.3 .2$ and, for each $x \in M_{o} \backslash S^{\prime}$, let us denote by $(g(x), t(x))$ some pair in $G \times \mathbb{R}$ with

$$
x=g(x) \cdot \eta_{t(x)} .
$$

By property (1) of $\eta$, such a pair surely exists, but is in general not unique. Indeed, $g(x)$ is determined up to composition with some $h \in L=G_{x}$, while $t(x)$ is unique in case $S^{\prime}$ is complex and determined up to a sign in all other cases. Then, we set

$$
\tau: M_{o} \backslash S^{\prime} \longrightarrow(0,+\infty), \quad \tau(x):= \begin{cases}e^{-t(x)} & \text { if } S^{\prime} \text { is complex }, \\ (t(x))^{2} & \text { if } S^{\prime} \text { is not complex } .\end{cases}
$$

By construction, $\tau$ is $G$-invariant. We claim it is also $\mathcal{C}^{\infty}$. By $G$-invariance, the claim is proven if we show that $\tau$ is smooth at each fixed $y_{0}:=\eta_{t_{o}}, 0<t_{o}<\infty$. For this, consider the decomposition $\mathfrak{g}=\mathfrak{l}+\mathbb{R} Z+\mathfrak{m}$, with $\mathfrak{l}:=\mathfrak{g}_{y_{o}}$, described in (3.3), and let $\mathfrak{g}_{y_{o}}^{\mathbb{C}} \subset \mathfrak{g}^{\mathbb{C}}$ be the isotropy subalgebra at $y_{o}$ of the complexified group $G^{\mathbb{C}}$. Further, denote by $\mathfrak{n}$ the $2 n$-dimensional real subspace $\mathfrak{n}:=\mathfrak{m}+\mathbb{R} Z+\mathbb{R}(i Z) \subset \mathfrak{g}^{\mathbb{C}}$, which is complementary to $\mathfrak{g}_{y_{o}}^{\mathbb{C}}$, and choose a real basis $\left(F_{1}, \ldots F_{2 n}\right)$ for $\mathfrak{n}$ with $F_{2 n}:=i Z$. We define $\widetilde{\exp }_{y_{o}}: \mathbb{R}^{2 n} \longrightarrow M_{o}$ by

$$
\widetilde{\exp }_{y_{o}}\left(y^{1}, \ldots, y^{2 n}\right):=\left(e^{y^{1} F_{1}} \cdots e^{y^{2 n-1} F_{2 n-1}} e^{y^{2 n} F_{2 n}}\right) \cdot y_{o}=\left(e^{y^{1} F_{1}} \cdots e^{y^{2 n-1} F_{2 n-1}}\right) \cdot \eta_{t_{o}+y^{2 n}} \text {. }
$$


Since $\mathfrak{n}$ is complementary to $\mathfrak{g}_{y_{o}}^{\mathbb{C}}$, the Jacobian at 0 at $\widetilde{\exp }_{y_{o}}$ is invertible, so that, by the Implicit Function Theorem, the map $\widetilde{\exp }_{y_{o}}$ gives a diffeomorphism between a neighborhood $\mathcal{V}$ of $0 \in \mathbb{R}^{2 n}$ and a neighborhood $\mathcal{U}$ of $y_{o} \in M_{o}$. We therefore have that the inverse

$$
\xi=\left(\widetilde{\exp }_{y_{o}}\right)^{-1}: \mathcal{U} \longrightarrow \mathcal{V} \subset \mathbb{R}^{2 n}
$$

is a system of real coordinates $\xi=\left(y^{1}, \ldots, y^{2 n}\right)$ near $y_{o}$. Since $e^{y^{1} F_{1}} \ldots e^{y^{2 n-1} F_{2 n-1}}$ is always an element of the real Lie group $G$, from (3.11) we see that for each element $y \in \mathcal{U}$ we may choose as $(g(y), t(y))$ the pair

$$
g(y)=e^{y^{1} F_{1}} \cdots e^{y^{2 n-1} F_{2 n-1}}, \quad t(y)=y^{2 n}+t_{o} .
$$

Hence, for each $y \in \mathcal{U}$,

$$
\tau(y)= \begin{cases}e^{-\left(y^{2 n}(y)+t_{o}\right)} & \text { if } S^{\prime} \text { is complex }, \\ \left(y^{2 n}(y)+t_{o}\right)^{2} & \text { if } S^{\prime} \text { is not complex }\end{cases}
$$

from which it follows immediately that $\left.\tau\right|_{\mathcal{U}}$ is of class $\mathcal{C}^{\infty}$, as desired.

We now want to show that for any given point $y \in S^{\prime}$, there exists a smooth extension of $\tau$ on a whole neighborhood of $y$, so that we may consider $\tau$ as a smooth real function over the whole $M_{o}$. The proof of this property is divided into two cases.

Case 1: the singular $G$-orbit $S^{\prime}$ is not complex. Under this assumption, either $M_{o}$ is a Morimoto-Nagano space or $M=M_{o} \cup S$ is an almost homogeneous manifold with one end and of the mixed type. Assume that the first holds, i.e. $M_{o}=T S^{\prime}$ for a CROSS of the form $S^{\prime}=G / K$. By 33.3.3, $M_{o} \simeq G \times_{K} \mathfrak{p}$ and $\eta_{t}$ is identifiable with a curve (3.8) for some unitary $Z \in \mathfrak{p}$. Then, by $G$-invariance of the norm $\|\cdot\|:=\sqrt{-\mathcal{B}(\cdot, \cdot)}$, for each $x=[(g, Y)]_{K} \in T S^{\prime} \backslash\{$ zero section $\}$, we may choose as $(g(x), t(x))$ the pair

$$
g(x):=g, \quad t(x):= \pm\|Y\|^{2} .
$$

This implies that the map $\tau: M_{o} \backslash S^{\prime}=G \times_{K}(\mathfrak{p} \backslash\{0\}) \rightarrow \mathbb{R}$ has the form

$$
\tau\left([(g, Y)]_{K}\right)=\|Y\|^{2}=-\mathcal{B}(Y, Y),
$$

which can be directly checked to be $\mathcal{C}^{\infty}$ over the entire $T S^{\prime} \simeq G \times_{K} \mathfrak{p} \simeq G^{\mathbb{C}} / K^{\mathbb{C}}$.

Assume now that $M=M_{o} \cup S$ is an almost homogeneous manifold of the third class. Then $M_{o}$ is a $G$-homogeneous bundle over a flag manifold $G / G_{Q}$, with fibers given by MorimotoNagano spaces. By $G$-invariance of $\tau$ and the fact that curve $\eta$ is entirely contained in a single fiber $\pi^{-1}\left(z_{o}\right), z_{o} \in G / G_{Q}$, the smooth extendibility of $\tau$ at the points of the singular $G$-orbit $S^{\prime}$ is equivalent to the smooth extendibility of the restriction of $\left.\tau\right|_{\pi^{-1}\left(z_{o}\right) \backslash S^{\prime}}$ to all points of $\pi^{-1}\left(z_{o}\right)$. And this is checked by the same above argument.

Case 2: the singular $G$-orbit $S^{\prime}$ is complex. In this situation, the manifold $M$ has two complex singular $G$-orbits $S, S^{\prime}$ and, since it is in canonical form, both of them have real codimension two. By $G$-invariance of the function $\tau$, there is no loss of generality if we assume that the point $y \in S^{\prime}$, around which we need to show that $\tau$ is smooth, coincides with the limit point $y=\lim _{t \rightarrow+\infty} \eta_{t}$. Let $K=G_{y}$ be the isotropy at $y$, so that the singular $G$-orbit $S^{\prime}$ is identifiable with $S^{\prime}=G / K$. Note that, by property (4) of $\eta$ and a dimensional argument, if $\mathfrak{g}=\mathfrak{l}+\mathfrak{m}+\mathbb{R} Z$ is the decomposition (3.3) corresponding to the regular point $x_{o}=\eta_{0} \in M_{o} \backslash S^{\prime}$, then $\mathfrak{k}=\mathfrak{l}+\mathbb{R} Z$.

Consider a $K$-invariant Kähler metric $g$ on $M$ and let $V=\left(T_{y} S^{\prime}\right)^{\perp}$ be the 2-dimensional $g$-orthogonal complement to $T_{y} S^{\prime}$ in $T_{y} M_{o}$. Since $S^{\prime}$ is complex, $V$ is $J$-invariant. Denote by $\exp _{y}: V \rightarrow M$ the restriction to $V$ of the exponential map of $(M, g)$ at $y$. 
By standard facts on proper actions (see e.g. [7, 12, 28]), the isotropy representation of $K$ on $T_{y} M$ preserves $V$ and acts linearly and isometrically on $V$ with codimension one regular orbits. It also preserves the complex structure $J_{o}=\left.J_{y}\right|_{V}$. Hence, we may identify $\left(V, J_{o},\left.g_{y}\right|_{V}\right)$ with $\left(\mathbb{C}, J_{o},\langle\cdot, \cdot\rangle\right)$, where $\langle\cdot, \cdot\rangle$ is the standard Euclidean product, so that the representation of $\rho: K \rightarrow \mathrm{GL}(V)$ is such that $\rho(K)=S^{1}$.

Consider the linear bundle $\pi: G \times_{K} \mathbb{C} \rightarrow S^{\prime}=G / K$. It is known that there exists a $K$-invariant neighborhood $\mathcal{U} \subset V$ of the origin such that the map

$$
\varphi: G \times_{K} \mathcal{U} \longrightarrow M_{o}, \quad \varphi\left([g, v]_{K}\right):=\exp _{g \cdot y}(v)=g \cdot \exp _{y}(v)
$$

is a $G$-equivariant diffeomorphism between $G \times{ }_{K} \mathcal{U}$ and a neighborhood $\mathcal{V}=\varphi\left(G \times{ }_{K} \mathcal{U}\right)$ of $S^{\prime}$ in $M_{o}$. By construction, the singular orbit $S^{\prime}$ coincides with the image by $\varphi$ of the zero section $G \times{ }_{K}\{0\}$ and the action of $e^{\mathbb{R} Z}$ on $\varphi(\mathcal{U})$ corresponds to the action on $G \times{ }_{K} \mathcal{U}$ defined by

$$
e^{t Z} \cdot[(g, \zeta)]_{K}:=\left[\left(g, e^{i t} \zeta\right)\right]_{K} .
$$

This yields that the infinitesimal transformations $\widehat{Z}, J \widehat{Z}=\widehat{(i Z)}$ determine on each fiber $\{g\} \times{ }_{K} \mathcal{U} \simeq \mathcal{U} \subset \mathbb{C}$ of $G \times{ }_{K} \mathcal{U}$ the (real) vector fields of $\mathbb{C} \simeq \mathbb{R}^{2}$

$$
\widehat{Z}=i \zeta \frac{\partial}{\partial \zeta}-i \bar{\zeta} \frac{\partial}{\partial \bar{\zeta}}, \quad J \widehat{Z}=-\zeta \frac{\partial}{\partial \zeta}+\bar{\zeta} \frac{\partial}{\partial \bar{\zeta}} .
$$

The flow of $J \widehat{Z}$ in $\{g\} \times{ }_{K} \mathcal{U} \simeq \mathcal{U} \subset \mathbb{C}$ is then given by

$$
\Phi_{t}^{J \widehat{Z}}(\zeta)=e^{-t}|\zeta|^{2}
$$

From this, we see that $\eta$ corresponds to the curve on $G \times{ }_{K} \mathcal{U}$

$$
\check{\eta}_{t}:=\varphi^{-1}\left(\eta_{t}\right)=\left[\left(e, e^{-t} \zeta_{o}\right)\right]_{K} \quad \text { where } \zeta_{o} \text { is such that }\left[\left(e, \zeta_{o}\right)\right]_{K}=\varphi^{-1}\left(x_{o}\right) \text {. }
$$

So, for each $x=\varphi\left([(g, \zeta)]_{K}\right), \zeta \neq 0$, in $\varphi(\mathcal{U})$, we may choose $(g(x), t(x))$ as the pair

$$
g(x)=g, \quad t(x)(\zeta)=-\frac{1}{2} \log \left(\frac{|\zeta|^{2}}{\left|\zeta_{o}\right|^{2}}\right) .
$$

Hence, for all points of $\mathcal{V} \backslash S^{\prime} \simeq \varphi\left(G \times_{K}(\mathcal{U} \backslash\{0\})\right)$, the function $\tau$ is such that

$$
\tau\left(\varphi\left([(g, \zeta)]_{K}\right)=C|\zeta|^{2} \quad \text { with } C:=\frac{e^{\frac{1}{2}}}{\left|\zeta_{o}\right|^{2}}\right.
$$

and it is clearly smoothly extendible at all points of $\mathcal{V} \simeq \varphi\left(G \times{ }_{K} \mathcal{U}\right)$, as claimed.

We now want to show that, in all cases, the map $\tau: M_{o} \rightarrow[0,+\infty)$ satisfies (1) and (2) of Definition 2.1. For this, consider the distributions $\mathcal{Z}, \mathcal{H} \subset T\left(M_{o} \backslash S^{\prime}\right)$, defined by

$$
\mathcal{Z}_{x}:=\left\langle\widehat{Z}_{x}, J \widehat{Z}_{x}\right\rangle, \quad \mathcal{H}_{x}:=\mathcal{D}_{x}, \quad x \in M_{o} \backslash S^{\prime},
$$

where $Z$ is the unitary element of $\mathfrak{g}$, appearing in (3.3), which is $\mathcal{B}$-orthogonal to the isotropy $\mathfrak{l}=\mathfrak{l}_{x}$ and to the space $\mathfrak{m} \simeq \mathcal{D}_{x}$, corresponding to the CR structure of the orbit $G \cdot x$. Note that such $Z \in \mathfrak{g}$ does depend on $x$ and, for the sake of clarity, it will be later denoted by $Z(x)$.

A direct check shows that $\mathcal{Z}$ and $\mathcal{H}$ are $d d^{c} \tau$-orthogonal and that, for each $x$, the restricted 2 -form $\left.d d^{c} \tau_{x}\right|_{\mathcal{H}_{x} \times \mathcal{H}_{x}}$ is (up to a multiple) the Levi form of the $G$-orbits $G \cdot x$, hence strictly positive. So, for (1) of Definition 2.1, we just need to show that $d d^{c} \tau \mid \mathcal{Z}_{x} \times \mathcal{Z}_{x}$ is positive at each $x \in M_{o} \backslash S^{\prime}$. For this, we first observe that the distribution $\mathcal{Z}$ is integrable and its integral leaves are the orbits in $M_{o} \backslash S^{\prime}$ of the complex Lie groups $\exp (\mathbb{C} Z(x))$. Hence

$$
\left.d d^{c} \tau\right|_{\mathcal{Z}_{x} \times \mathcal{Z}_{x}}=\left.d d^{c}\left(\left.\tau\right|_{\exp (\mathbb{C} Z(x)) \cdot x}\right)\right|_{x} .
$$


By previous discussion,

$$
\left.\tau\right|_{\exp (\zeta Z(x)) \cdot x}=C|\zeta|^{2} \quad \text { or }\left.\quad \tau\right|_{\exp (\zeta Z(x)) \cdot x}=C(\operatorname{Im} \zeta)^{2},
$$

the first occurring when $M=M_{o} \cup S$ has two complex singular $G$-orbits, the second in all other cases. From (3.13) and (3.14), we get $d d^{c} \tau_{x} \mid \mathcal{Z}_{x} \times \mathcal{Z}_{x}>0$ in all cases, as desired.

In order to check (2) of Definition 2.1, we first observe that for any smooth function $f:(0,+\infty) \rightarrow \mathbb{R}$ with nowhere vanishing differential $d f$, we have $\left.d d^{c}(f \circ \tau)\right|_{\mathcal{H}_{x} \times \mathcal{H}_{x}}>0$ at each point $x$. Therefore, by (3.13), property (2) holds if and only if there is such an $f$ with harmonic restrictions $\left.f \circ \tau\right|_{\exp (\mathbb{C} Z(x))}$. By (3.14), we see that $f(t)=\log (t)$ and $f(t)=\sqrt{t}$ satisfy the request in the two cases. This concludes the proof of (i).

It remains to prove (ii). Let $\tau^{\prime}: M_{o} \rightarrow[0,+\infty)$ be a $G$-invariant Monge-Ampère exhaustion of $M_{o}$ satisfying $(\alpha),(\beta)$ and $(\gamma)$. Since $d d^{c} \tau^{\prime}$ and $d d^{c}\left(\log \circ \tau^{\prime}\right)$ are both positive on the CR distributions of the regular $G$-orbits (which are level sets of $\tau^{\prime}$ and $f \circ \tau^{\prime}$ and are strongly pseudoconvex), due to $(\alpha)$ the restrictions $\left.\log \circ \tau^{\prime}\right|_{\exp (\mathbb{C} Z(x)) \cdot x}$ or $\left.\sqrt{\tau^{\prime}}\right|_{\exp (\mathbb{C} Z(x)) \cdot x}$ are necessarily harmonic. They are also constant along the sets $\exp (\mathbb{R} Z(x)) \cdot x$, which are the intersections of $(\exp (\mathbb{C} Z(x)) \cdot x)$ with the $G$-orbits.

Let us first prove that all this implies (ii) when $M=M_{o} \cup S$ has two ends. By the proof of (i), we know that each orbit $\exp (\mathbb{C} Z(x)) \cdot x$ is identifiable with $\mathbb{C} \backslash\{0\}$, so that the group $\{\exp (\zeta Z(x)), \zeta \in \mathbb{C}\}$ corresponds to the group $\left\{D_{\zeta}(z):=e^{i \zeta} \cdot z, \zeta \in \mathbb{C}\right\}$. Under such identification, the restriction $\left.\log \circ \tau^{\prime}\right|_{\exp (\mathbb{C} Z(x)) \cdot x}$ is an harmonic function of $\mathbb{C} \backslash\{0\}$ depending only on the distance from the origin. It has therefore the form

$$
\begin{aligned}
& \log \circ \tau^{\prime}(\exp (\zeta Z(x)) \cdot x)=a_{x}+2 b_{x} \log (|\zeta|) \quad \text { for some constant } a_{x}, b_{x} \in \mathbb{C}, \text { so that } \\
& \tau^{\prime}(\exp (\zeta Z(x)) \cdot x)=c_{x}\left(|\zeta|^{2}\right)^{b_{x}} \quad \text { with } c_{x}:=e^{a_{x}} .
\end{aligned}
$$

Condition $(\gamma)$ and $G$-invariance imply that $b_{x} \equiv 1$ and that the constant $c_{x}$ does not depend on $x$. From (3.14), (ii) follows in this case.

We now prove (ii) holds when $M$ has only one end. In this case, each orbit $\exp (\mathbb{C} Z(x)) \cdot x$ is identifiable with a quotient $\mathbb{C} / \Gamma$ with $\Gamma$ group of real translations $\Gamma=\left\{T_{k}(z):=z+\right.$ $2 \pi k \zeta, k \in \mathbb{Z}\}$. Under this identification, the group $\{\exp (\zeta Z(x))$ acts on such orbit as the group of complex translations $\{T(z):=z+\zeta, \zeta \in \mathbb{C}\}$ and the restriction $\sqrt{\tau^{\prime}} \mid \exp (\mathbb{C} Z(x)) \cdot x$ is identified with an harmonic function of $\mathbb{C} / \Gamma$, which is constant on the lines $\{\operatorname{Im}(z)=c\}$, $c \in \mathbb{R}$. Hence

$$
\sqrt{\tau^{\prime}(\exp (\zeta Z(x)) \cdot x)}=a_{x}+2 b_{x} \operatorname{Im}(\zeta) \quad \text { for some constant } a_{x}, b_{x} \in \mathbb{C} .
$$

Condition $(\gamma)$ and $G$-invariance imply that $a_{x} \equiv 0$ and that the constant $b_{x}$ does not depend on $x$. From (3.14), claim (ii) follows also in this case.

\section{Deformability versus Rigidity}

As we already mentioned, Theorem 3.2 is a source of several new examples of MongeAmpère spaces. On the other hand, the previously known examples include two important families of Monge-Ampère spaces, the manifolds of circular type and the Grauert tubes, having the following contrasting properties: the first can be all considered as deformations of $\mathbb{C}^{n}$ or $\mathbb{B}^{n}([24,26])$, while the second are characterized by strong rigidity results $([18])$. Motivated by this, in this final section we investigate whether also the new examples enjoy manifest deformability (or rigidity) properties. Let us begin by fixing the meaning of "deformability" for a Monge-Ampère space. 


\subsection{Riemann mappings and deformations of modeling spaces.}

Let $\mathcal{X}$ be a Monge-Ampère space, which is a Remmert reduction $\pi: \widetilde{\mathcal{X}} \rightarrow \mathcal{X}$ of a complex manifold $\widetilde{\mathcal{X}}$ with Monge-Ampère exhaustion $\tau: \widetilde{\mathcal{X}} \rightarrow[0, T)$. Let also $u=f \circ \tau: \widetilde{S} \rightarrow \mathbb{R}$ be function satisfying the conditions $2 i \partial \bar{\partial} u \geq 0$ and $(\partial \bar{\partial} u)^{n}=0$, and $\mathcal{S}=\pi\left(\tau^{-1}(0)\right)$ the soul of $\mathcal{X}$. We recall that $\mathcal{X} \backslash \mathcal{S}$ is a complex manifold, naturally identifiable with $\widetilde{\mathcal{X}} \backslash \tau^{-1}(0)$, so that both exhaustions $\tau$ and $u$ are well defined and smooth on $\mathcal{X} \backslash \mathcal{S}$.

We now observe that all local properties of exhaustions $\tau$ and $u$ on the manifold $\mathcal{X} \backslash \mathcal{S}$, which have been proven in the literature for some specific cases (as, for instance, when $\mathcal{X}$ is a manifold of circular type or a Morimoto-Nagano spaces - see e.g. [34, 23, 25, 27]) are valid for any Monge-Ampère space $\mathcal{X}$. In particular, one can directly check that there is always a well defined vector field $Z$ on $\mathcal{X} \backslash \mathcal{S}$ that satisfies the condition

$$
d d^{c} \tau(J Z, J X)=X(\tau) \quad \text { for any vector field } X \in T(\mathcal{X} \backslash \mathcal{S}) .
$$

For the spaces of Theorem [3.2, this vector field verifies $Z_{x}= \pm \widehat{Z(x)}$ at each $x \in M_{o} \backslash S^{\prime}$. By $J$-invariance of the 2 -form $d d^{c} \tau$ and integrability of the complex structure $J$, the vector field $Z$ is tangent to each level set $\tau^{-1}(c), c \in(0, T)$, and $Z$ and $J Z$ generate a $J$-invariant, integrable 2-dimensional distribution $\mathcal{Z} \subset T(\mathcal{X} \backslash \mathcal{S})$, called Monge-Ampère distribution. A direct computation shows that it coincides with the distribution defined by $\mathcal{Z}_{x}=$ ker $\left.d d^{c} u\right|_{x}$ at each $x \in \mathcal{X} \backslash \mathcal{S}$.

The foliation $\mathcal{F}$, given by the integral leaves of $\mathcal{Z}$, is called the Monge-Ampère foliation of $(\mathcal{X}, \tau)$. The closures in $\mathcal{X}$ of these leaves sometimes form a regular foliation, sometimes not. However, in all known examples, the closures of their lifts on the manifold $\widetilde{\mathcal{X}}$ always form a regular foliation of $\widetilde{\mathcal{X}}$. For brevity, when this property occurs we say that the Monge-Ampère foliation is $\widetilde{\mathcal{X}}$-regular. For the spaces in Theorem 3.2 , the Monge-Ampère foliation $\mathcal{F}$ consists of orbits in $M_{o} \backslash S^{\prime}$ of the 1-dimensional complex groups $\exp (\mathbb{C} Z(x))$ and, in all such cases, $\mathcal{F}$ is $M_{o}$-regular.

It is clear that if $\varphi: \mathcal{X} \rightarrow \mathcal{X}^{\prime}$ is a biholomorphism between two Monge-Ampère spaces $(\mathcal{X}, \tau),\left(\mathcal{X}^{\prime}, \tau^{\prime}\right)$ with $\tau=\tau^{\prime} \circ \varphi$, then $\varphi$ maps biholomorphically each leaf of the MongeAmpère foliation foliation of $\mathcal{X}$ into a corresponding leaf of the Monge-Ampère foliation of $\mathcal{X}^{\prime}$. In certain cases this property admits an inverse, in the sense that if $\varphi: \mathcal{X} \rightarrow \mathcal{X}^{\prime}$ is an homeomorphism with $\tau=\tau^{\prime} \circ \varphi$ and mapping biholomorphically each Monge-Ampère leaf of $\mathcal{X}$ into a corresponding leaf of $\mathcal{X}^{\prime}$, then $\varphi$ is a biholomorphism provided that certain additional hypothesis are satisfied. Stoll's characterization of $\mathbb{C}^{n}$ and Lempert and Szöke rigidity theorems for Grauert tubes can be considered as examples of such kind of property. All this motivates the next notion.

Consider a fixed Monge-Ampère space $\left(\mathcal{X}_{o}, \tau_{o}\right)$, which we call model from now on. Let also $\pi: \widetilde{\mathcal{X}}_{o} \rightarrow \mathcal{X}_{o}$ be the Remmert reduction that determines the Monge-Ampère space $\mathcal{X}_{o}$ and denote by $\mathcal{S}_{o}$ and $\mathcal{F}_{o}$ the soul and the Monge-Ampère foliation of $\mathcal{X}_{o}$, respectively. We assume that $\mathcal{F}_{o}$ is $\widetilde{\mathcal{X}}_{o}$-regular, as it occurs in all considered examples.

Definition 4.1. Let $(\mathcal{X}, \tau)$ be a Monge-Ampère space, Remmert reduction of a complex manifold $\widetilde{\mathcal{X}}$, with soul $\mathcal{S}$ and $\widetilde{\mathcal{X}}$-regular Monge-Ampère foliation $\mathcal{F}$. We say that $(\mathcal{X}, \tau)$ is modeled on $\left(\mathcal{X}_{o}, \tau_{o}\right)$ if there is a homeomorphism $\varphi: \mathcal{X}_{o} \rightarrow \mathcal{X}$ such that $\tau_{o}=\tau \circ \varphi$ and:

i) $\left.\varphi\right|_{\mathcal{X}_{o} \backslash \mathcal{S}_{o}}: \mathcal{X}_{o} \backslash \mathcal{S}_{o} \rightarrow \mathcal{X} \backslash \mathcal{S}$ is a diffeomorphism mapping biholomorphically each leaf of $\mathcal{F}_{o}$ into a leaf of $\mathcal{F}$;

ii) the restriction $\left.\varphi\right|_{\mathcal{X}_{o} \backslash \mathcal{S}_{o}}$ lifts to a diffeomorphism $\tilde{\varphi}: \widetilde{\mathcal{X}}_{o} \backslash \pi^{-1}\left(\mathcal{S}_{o}\right) \rightarrow \widetilde{\mathcal{X}} \backslash \pi^{-1}(\mathcal{S})$ which smoothly extends to a diffeomorphism between the $\widetilde{\mathcal{X}}_{o}$ and $\widetilde{\mathcal{X}}$. 
Any such homeomorphism $\varphi: \mathcal{X}_{o} \rightarrow \mathcal{X}$ is called Riemann mapping of $\mathcal{X}$. The spaces that are modeled on $\left(\mathcal{X}_{o}, \tau_{o}\right)$, but are not biholomorphic to $\mathcal{X}_{o}$, are called non-trivial deformations of the model.

\subsection{Soul rigidity, soul semi-rigidity and free deformability.}

Looking at the known examples, there are models with a lot of non-trivial deformations and others with no deformation with sufficiently regular Riemann mappings. For instance, the results in [5, 16, 24, show that any smoothly bounded strictly convex domain in $\mathbb{C}^{n}$, not biholomorphic to $\mathbb{B}^{n}$, is a non-trivial deformation of the standard unit ball $\left(\mathbb{B}^{n}, J_{o},\|\cdot\|^{2}\right)$. On the other hand, Stoll's characterization of $\mathbb{C}^{n}([34,8,26])$ shows that there exists no nontrivial deformations of $\left(\mathbb{C}^{n}, J_{o},\|\cdot\|^{2}\right)$ in the class of Monge-Ampère manifolds with Riemann mappings of class $\mathcal{C}^{2}$. Known rigidity results for Grauert tubes give uniqueness for the Riemann mappings from Morimoto-Nagano spaces ([13, 18, 27]). For clarifying similarities and differences between all such results, we now introduce the following notions.

Let $\varphi: \mathcal{X}_{o} \rightarrow \mathcal{X}$ be a Riemann mapping from a model $\mathcal{X}_{o}$ and denote by $\widetilde{\varphi}: \widetilde{\mathcal{X}}_{o} \rightarrow \widetilde{\mathcal{X}}$ the corresponding lifted diffeomorphism between the manifolds, which project onto the two spaces by Remmert reductions $\pi_{o}: \widetilde{\mathcal{X}}_{o} \rightarrow \mathcal{X}_{o}$ and $\pi: \widetilde{\mathcal{X}} \rightarrow \mathcal{X}$. Denoting by $J_{o}, J$ the complex structures of $\widetilde{\mathcal{X}}_{o}, \widetilde{\mathcal{X}}$, respectively, the diffeomorphism $\widetilde{\varphi}$ is a biholomorphism if and only if for any tangent vector $v \in T_{x} \widetilde{\mathcal{X}}_{o}, x \in \widetilde{\mathcal{X}}_{o}$,

$$
\varphi_{*}\left(J_{o}(v)\right)=J \varphi_{*}(v) .
$$

It is now convenient to consider the following weaker conditions. We say that $\varphi$ is:

1) a biholomorphism at the blow ups of the souls if it satisfies (4.2) for any vector $v \in T_{x} \pi_{o}^{-1}\left(\mathcal{S}_{o}\right)$ in a tangent space of the preimage $\pi^{-1}\left(\mathcal{S}_{o}\right)$ of the soul $\mathcal{S}_{o}$;

2) a biholomorphism between the souls if it satisfies (4.2) for any vector $v \in T_{x} \pi_{o}^{-1}\left(\mathcal{S}_{o}\right)$ in a tangent space of $\pi^{-1}\left(\mathcal{S}_{o}\right)$ which projects onto a non trivial tangent vector of $\mathcal{S}_{o}$.

The second condition is manifestly weaker than the first since it does not requires that (4.2) holds for vectors that are in $\left.\operatorname{ker} \pi_{*}\right|_{y}, y \in \pi^{-1}\left(\mathcal{S}_{o}\right)$. Moreover, note that:

a) When $\mathcal{X}_{o}$ is the Remmert reductions of some $M_{o}=M \backslash S$, with $M$ almost homogeneous with two ends, condition (2) is trivial, since in this case the soul is an isolated point and there are no non-trivial tangent vector for such soul.

b) When $\mathcal{X}_{o}, \mathcal{X}$ are Grauert tubes over two Riemannian manifolds $\left(M_{o}, g_{o}\right),(M, g)$, respectively, condition (2) coincides with condition (1) and it is equivalent to require that the Riemann mapping $\varphi$ induces an isometry between $\left(M_{o}, g_{o}\right)$ and $(M, g)$.

c) When $\mathcal{X}_{o}$ and $\mathcal{X}$ are manifolds of circular type, as e.g. two strictly convex domains in $\mathbb{C}^{n},(2)$ is a trivial condition because of (a), while (1) is equivalent to require that the Riemann mapping induces an isometry between the Kobayashi indicatrices at the centers.

We now say that a model $\mathcal{X}_{o}$ is

- soul rigid if any Riemann mapping $\varphi: \mathcal{X}_{o} \rightarrow \mathcal{X}$ which is a biholomorphism between the souls is a biholomorphism between the two Monge-Ampère spaces;

- soul semi-rigid if it is not soul rigid, but nonetheless for any Riemann mapping $\varphi$ : $\mathcal{X}_{o} \rightarrow \mathcal{X}$ which is a biholomorphism at the blow ups of the souls is a biholomorphism between the two Monge-Ampère spaces;

- fully deformable if it is not of the previous two types. 
The quoted rigidity results for Morimoto-Nagano spaces and Grauert tubes can be stated saying that those manifolds are soul rigid. On the other hand, the results of [8, 23] show that $\left(\mathbb{C}^{n},\|\cdot\|^{2}\right)$ is soul semi-rigid, while the examples in [5, 24, 26] show that the standard unit ball $\left(\mathbb{B}^{n}, J_{o},\|\cdot\|^{2}\right)$ is a fully deformable model with a lot of non-trivial deformations.

\subsection{New examples of semi-rigid and fully deformable models.}

The following theorem gives a common framework for the so far known rigidity results on Monge-Ampère spaces. It also indicates a new interesting class of semi-rigid examples and, by the examples in 4.3 .2 below, suggests the existence of a large new family of fully deformable Monge-Ampère spaces.

Theorem 4.2. Let $M$ be one of the almost homogeneous manifolds in canonical form, described in $\S \S$ 3.2.1, 3.2.2, 3.2.3, and $\tau_{o}: M_{o}=M \backslash S \rightarrow[0,+\infty)$ the Monge-Ampère exhaustion of Theorem [3.2. Let also $\mathcal{M}_{o}$ be the corresponding Remmert reduction of $M_{o}$, equipped with the exhaustion induced by $M_{o}$, which, for simplicity of notation, we also denote by $\tau_{o}$. For any $c \in(0,+\infty]$, let $\mathcal{M}_{o}(c):=\left\{x: \tau_{o}(x)<c\right\}$ be the Monge-Ampère subspace with exhaustion $\left.\tau_{o}\right|_{\mathcal{M}_{o}(c)}$. Then:

i) if $M$ has two ends, the space $\left(\mathcal{M}_{o}(\infty), \tau_{o}\right)=\left(\mathcal{M}_{o}, \tau_{o}\right)$ is soul semi-rigid; on the other hand, there is an $M$ with two ends for which all Monge-Ampère spaces $\left(\mathcal{M}_{o}(c), \tau_{o}\right)$ with $c<\infty$ are fully deformable;

ii) if $M$ is a compactification of a Morimoto-Nagano space, each Monge-Ampère space $\left(\mathcal{M}_{o}(c), \tau_{o}\right), 0<c \leq \infty$, is soul rigid;

iii) if $M$ has one end and is of mixed type, then each Monge-Ampère space $\left(\mathcal{M}_{o}(c), \tau_{o}\right)$, $0<c \leq+\infty$, is soul semi-rigid.

The proof is crucially based on some properties of "deformation tensors" of certain complex structures and on some known counterexamples to soul rigidity or semi-rigidity. Before proving this theorem, we need to review such results in some detail.

4.3.1. Deformation tensors of deformed Monge-Ampère spaces. As usual, let $\mathcal{X}$ be a MongeAmpère space, which is Remmert reduction of a complex manifold $\widetilde{\mathcal{X}}$ with Monge-Ampère exhaustion $\tau: \widetilde{\mathcal{X}} \rightarrow[0, T)$. Denoting by $\mathcal{S}$ the soul of $\mathcal{X}$, we observe that on $\mathcal{X} \backslash \mathcal{S}$ (which always identifiable with the complementary set $\widetilde{\mathcal{X}} \backslash \pi^{-1}(\mathcal{S})$ ) one can consider the $J$-invariant distribution $\mathcal{H}$, called normal distribution, defined by

$$
\mathcal{H}_{x}=\left\{X \in T_{x} M: d d^{c} \tau(Z, X)_{x}=d d^{c} \tau(J Z, X)_{x}=0\right\} .
$$

By non-degeneracy of $d d^{c} \tau$ on $\mathcal{X} \backslash \mathcal{S}$, we have that $T_{x} M=\mathcal{Z}_{x} \oplus \mathcal{H}_{x}$ at each $x \in \mathcal{X} \backslash \mathcal{S}$. Moreover, for each level set $\tau^{-1}(c), c \in(0, T)$, the restriction $\left.\mathcal{H}\right|_{\tau^{-1}(c)}$ coincides with the $J$-invariant distribution of the induced CR structure $\left(\mathcal{D}=\left.\mathcal{H}\right|_{\tau^{-1}(c)}, J\right)$ of such level set.

Consider now a Monge-Ampère space $(\mathcal{X}, \tau)$ modeled on a manifolds $\left(\mathcal{X}_{o}, \tau_{o}\right)$, and denote by $J_{o}, J$ the complex structures of the complex manifolds $\widetilde{\mathcal{X}}_{o}, \widetilde{\mathcal{X}}$, of which $\mathcal{X}_{o}$ and $\mathcal{X}$ are Remmert reductions, respectively. Fix also a Riemann mapping $\varphi: \mathcal{X}_{o} \rightarrow \mathcal{X}$, with associated lifted diffeomorphism $\widetilde{\varphi}: \widetilde{\mathcal{X}}_{o} \rightarrow \widetilde{\mathcal{X}}$. As direct consequence of definitions, $\widetilde{\varphi}$ sends the Monge-Ampère and normal distributions of $\mathcal{X}_{o} \backslash \mathcal{S}_{o}$ into the corresponding distributions of $\mathcal{X} \backslash \mathcal{S}$.

We denote by $\mathbb{J}:=\widetilde{\varphi}_{*}^{-1}(J)$ the pull-back on $\mathcal{X}_{o}$ of the complex structure $J$. Clearly $\varphi$ is a biholomorphism if and only if $J_{o}=\mathbb{J}$.

We recall that, being a Riemann mapping, the map $\widetilde{\varphi}$ is a biholomorphism along each leaf of the Monge-Ampère foliation. This mean that $\left.J_{o}\right|_{\mathcal{Z}}=\left.\mathbb{J}\right|_{\mathcal{Z}}$ and that differences between $J_{o}$ 
and $\mathbb{J}$ might occur only when they are restricted on the normal distribution $\mathcal{H}$. On the other hand, at each point $x$, both complex structures $\left.J_{o}\right|_{\mathcal{H}_{x}},\left.\mathbb{J}\right|_{\mathcal{H}_{x}}: \mathcal{H}_{x} \rightarrow \mathcal{H}_{x}$ are determined by their $(-i)$-eigenspaces in the complexification $\mathcal{H}_{x}^{\mathbb{C}}$. Let us denote these eigenspaces by $\mathcal{H}_{x}^{01}$ and $\mathcal{H}_{x}^{\prime 01}$, respectively, and indicate by $\mathcal{H}^{01}, \mathcal{H}^{\prime 01} \subset \mathcal{H}^{\mathbb{C}}$ the involutive complex distributions, determined by such $(-i)$-eigenspaces. Their conjugate distributions (which are generated by the $(+i)$-eigenspaces $)$ are denoted by $\mathcal{H}^{10}=\overline{\mathcal{H}^{01}}$ and $\mathcal{H}^{\prime 10}=\overline{\mathcal{H}^{\prime 01}}$.

We now observe that, for a fixed $x$, if the standard projection $p: \mathcal{H}_{x}^{\mathbb{C}}=\mathcal{H}_{x}^{10}+\mathcal{H}_{x}^{01} \rightarrow \mathcal{H}_{x}^{01}$ satisfies the condition $p\left(\mathcal{H}_{x}^{\prime 01}\right)=\mathcal{H}_{x}^{01}$, then the space $\mathcal{H}_{x}^{\prime 01}$ has the form

$$
\mathcal{H}_{x}^{\prime 01}=\left\{v=w+\phi_{x}(w), w \in \mathcal{H}_{x}^{01}\right\}
$$

for some appropriate tensor $\phi_{x} \in\left(\mathcal{H}_{x}^{01}\right)^{*} \otimes \mathcal{H}_{x}^{10}$. The set $\mathcal{U} \subset \mathcal{X}(c)$ of points $x$, for which the condition $p\left(\mathcal{H}_{x}^{\prime 01}\right)=\mathcal{H}_{x}^{01}$ holds, is open and we call it the regularity set of $\mathbb{J}$. The tensors $\phi_{x}$ combine to a smooth tensor field $\phi$ on $\mathcal{U}$, called the deformation tensor of $\mathbb{J}([\underline{5}, 24,26])$.

The biholomorphicity condition can be now expressed in terms of the deformation tensor $\phi$ saying that $\varphi$ is a biholomorphism if and only if the regularity set $\mathcal{U}$ of $\mathbb{J}$ coincides with $\widetilde{\mathcal{X}}_{o}$ and $\phi \equiv 0$.

We conclude this section recalling a crucial property of the distributions $\mathcal{Z}$ and $\mathcal{H}$. We remark that the normal distribution $\mathcal{H}$ is invariant under the flows of the vector fields $Z$ and $J Z\left(=\mathbb{J} Z\right.$ ), defined in (4.1). Due to this and the fact that $\mathcal{Z}^{01}+\mathcal{H}^{\prime 01}$ is involutive (due to the integrability of $\mathbb{J})$, for each vector field in $\left.Y \in \mathcal{H}^{01}\right|_{\mathcal{U}}$

$$
\left[Z^{01}, Y+\phi(Y)\right]=\left[Z^{01}, Y\right]+\phi\left(\left[Z^{01}, Y\right]\right), \quad \text { where } Z^{01}:=Z+i J Z .
$$

So, if we denote by $L$ an integral complex leaf of $\mathcal{Z}$ and by $\left(e_{\alpha}, e_{\bar{\beta}}:=\overline{e_{\beta}}\right)$ is a frame field for $\mathcal{H}^{\mathbb{C}}=\mathcal{H}^{10}+\mathcal{H}^{01}$ on a neighborhood of $L$, invariant under the complex flows of $Z$, and if $\zeta$ is a complex coordinate on $L$ with $\frac{\partial}{\partial \zeta}=(Z-i J Z)=\overline{Z^{01}}$, then (4.5) yields that the components $\left.\phi_{\bar{\alpha}}^{\beta}\right|_{L}$ of $\left.\phi\right|_{L}$ in the frame $\left(e_{\alpha}, e_{\bar{\beta}}\right)$ are holomorphic functions of $\zeta$, i.e.

$$
\left.\frac{\partial \phi_{\bar{\alpha}}^{\beta}}{\partial \bar{\zeta}}\right|_{x}=0 \quad \text { at each } x \in L \text {. }
$$

4.3.2. Counterexamples to soul rigidity and soul semi-rigidity. Assume that $M$ is an almost homogeneous manifold as in (i) or (iii) of Theorem 4.2. Then $M$ is a fiber bundle $p: M \rightarrow G / K$ over a flag manifold $\left(G / K, J_{G / K}\right)$, with fiber $F$ equal either to $\mathbb{C} P^{1}$ or to a compactification of a Morimoto-Nagano space. In all these cases, the projection $p$ is holomorphic with respect to the $G$-invariant complex structures $J$ of $M$ and $J_{G / K}$ of $G / K$. Now, given an open set $\mathcal{U} \subset G / K$, for which there exists a holomorphic trivialization $p^{-1}(\mathcal{U}) \simeq \mathcal{U} \times F$, we may consider a non-trivial non-biholomophic diffeomorphism $h: G / K \rightarrow G / K$ mapping $\mathcal{U}$ into itself and satisfying the triviality condition $\left.h\right|_{G / K \backslash \mathcal{U}}=\left.\operatorname{Id}\right|_{G / K \backslash \mathcal{U}}$ on the complement of $\mathcal{U}$. Since $p^{-1}(\mathcal{U})$ is holomorphically trivializable, we may construct a fiber preserving diffeomorphism $\widetilde{\varphi}: M \rightarrow M$ such that:

A) it projects onto $h$ and is such that $\left.\widetilde{\varphi}\right|_{M \backslash p^{-1}(\mathcal{U})}=\operatorname{Id}_{M \backslash p^{-1}(\mathcal{U})}$;

B) it is holomorphic on each fibre of $p: M \rightarrow G / K$ and maps the level sets $\tau^{-1}(c) \cap F_{x}$ of each fiber $F_{x}=p^{-1}(x), x \in \mathcal{U}$, into the corresponding level sets $\tau^{-1}(c) \cap F_{h(x)}$ of the fiber $F_{h(x)}=\pi^{-1}(h(x))$.

Since all fibers $F_{x}, x \in \mathcal{U}$, are identifiable one to the other by means of a holomorphic trivialization $\pi^{-1}(\mathcal{U}) \simeq \mathcal{U} \times F$, a map that satisfies (A) and (B) can be easily determined. 
By construction, such diffeomorphism $\widetilde{\varphi}: M \rightarrow M$ leaves invariant all submanifolds $M_{o}(c):=\pi^{-1}\left(\mathcal{M}_{o}(c)\right), c \in(0,+\infty]$, and its restrictions to them are all Riemann mappings. But no such restriction is a biholomorphism. On the other hand, by construction, it is a biholomorphism between the souls, since it satisfies (4.2) for any vector $v \in T_{\pi^{-1}(x)}\left(\pi^{-1}\left(\mathcal{S}_{o}\right)\right)$ which projects onto some non trivial tangent vectors of the soul $\mathcal{S}_{o}$, this being diffeomorphic to each of the zero level sets $\tau^{-1}(0) \cap F_{x}$ of all fibers of the fibration $p: M \rightarrow G / K$

Assume now that $M$ is the blow up $p: \widetilde{\mathbb{C} P^{n}} \rightarrow \mathbb{C} P^{n}$ of $\mathbb{C} P^{n}$ at some point $x_{o}$. As we already mentioned, this is an almost homogeneous manifold with two ends, acted on by $G=\mathrm{SU}_{n}$ and such that the manifold $M_{o}=M \backslash S$ is naturally identifiable with the blow up $p: \widetilde{\mathbb{C}^{n}} \rightarrow \mathbb{C}^{n}$ of $\mathbb{C}^{n}$ at the origin. In this case, the Monge-Ampère spaces $\left(\mathcal{M}_{o}(c), \tau\right)$, $0<c<+\infty$, are identifiable with the balls $\mathbb{B}_{c}^{n} \subset \mathbb{C}^{n}$ of radius $c$ and center at 0 , equipped with the standard exhaustion $\|\cdot\|^{2}$. By [26], Cor. 6.2 and Thm. 5.2, we know that there are Monge-Ampère spaces, biholomorphic to strictly convex domains, which are non-trivial deformations of $\left(\mathbb{B}_{c}^{n},\|\cdot\|^{2}\right)$ with Riemann mappings that induce the identity map on the preimages of the souls. This means that all such Monge-Ampère spaces are fully deformable models. We expect that this property holds for all models $\left(\mathcal{M}_{o}(c), \tau\right), 0<c<+\infty$, that are determined by the almost homogeneous manifolds with two ends in canonical form.

4.3.3. Proof of Theorem 4.2. Let $\varphi: \mathcal{M}_{o}(c) \rightarrow \mathcal{X}$ be a Riemann mapping between one of the models $\left(\mathcal{M}_{o}(c), \tau_{o}\right)$ considered in (i) - (iii) and a Monge-Ampère space $(\mathcal{X}, \tau)$. As usual, we denote by $\widetilde{\varphi}: M_{o}(c) \rightarrow \widetilde{\mathcal{X}}$ the associated lifted diffeomorphism and by $\mathbb{J}=\widetilde{\varphi}_{*}^{-1}(J)$ the pull-back on $M_{o}(c)$ of the complex structure $J$ of $\widetilde{\mathcal{X}}$. We first want to prove that, for all models in (ii) and (iii), if $\varphi$ satisfies (4.2) at all tangent spaces of $\pi^{-1}\left(\mathcal{S}_{o}\right)$, then the regular set $\mathcal{U}$ of $\mathbb{J}$ coincides with the whole $M_{o}(c)$ and the corresponding deformation tensor $\phi$ vanishes identically, meaning that $\varphi$ is a biholomorphism.

We recall that the distributions $\mathcal{Z}$ and $\mathcal{H}$ are both $J_{o}$ and $\mathbb{J}$-invariant and that the complex structures $J_{o}$ and $\mathbb{J}$ agree on the vector fields in $\mathcal{Z}$. Note also that for each $\mathcal{M}_{o}(c)$, the $J_{o}$-invariant distributions $\mathcal{Z}$ and $\mathcal{H}$, taken as distributions on $M_{o}(c) \backslash \pi^{-1}(\mathcal{S})=M_{o}(c) \backslash S^{\prime}$, extend smoothly at all points of $M_{o}(c)$. These property imply that $\varphi$ satisfies (4.2) at all tangent spaces of $\pi^{-1}\left(\mathcal{S}_{o}\right)$ if and only if $\left.J_{o}\right|_{\mathcal{H}_{x}}=\left.\mathbb{J}\right|_{\mathcal{H}_{x}}$ at all points $x \in \pi^{-1}\left(\mathcal{S}_{o}\right)=S^{\prime}$. If this is the case, then the regular set $\mathcal{U}$ of the complex structure $\mathbb{J}$ clearly includes the submanifold $\pi^{-1}\left(\mathcal{S}_{o}\right)=S^{\prime}$, the deformation tensor $\phi$ of $\mathbb{J}$ is well defined on a tubular neighborhood $\mathcal{W}$ of $\pi^{-1}\left(\mathcal{S}_{o}\right)$ and the restriction $\left.\phi\right|_{\pi^{-1}\left(\mathcal{S}_{o}\right)}$ is identically equal to 0 .

Assume now that $M=M_{o} \cup S$ is either a compactification of a Morimoto-Nagano manifold or an almost homogeneous manifold with one end and of mixed type. In these two cases, the leaves of the Monge-Ampère foliation of $M_{o}(c) \subset M_{o}$ are (contained in) orbits of the 1-dimensional Lie groups $\exp (\mathbb{C} Z(x))$ described in 4.1 , and intersect the pre-image $\pi^{-1}\left(\mathcal{S}_{o}\right)=S^{\prime}$ of the soul along sets, which have Hausdorff dimension 1. This fact, together with the holomorphicity of the components of $\phi$ in the complex coordinate $\zeta$ of each leaf $L=\{\exp (\zeta Z(x)) \cdot x, \zeta \in \mathbb{C}\}$, implies that if the Riemann mapping $\varphi$ is a biholomorphism at the blow ups of the souls, then the restriction $\left.\phi\right|_{L}$ is identically equal to 0 along each such leaf $L$. This means that the regular set $\mathcal{U}$ of $\mathbb{J}$ contains all leaves of the Monge-Ampère foliation, hence the whole $\mathcal{U}=M_{o}(c)$, and that $\phi$ vanishes identically on $M_{o}(c)$ as claimed.

We claim that the same conclusion holds also if $M$ is as in (i) and the considered model is $\left(M_{o}(c), \tau\right)$ with $c=\infty$. In this case the closure in $M_{o}(\infty)=M_{o}$ of a leaf $L$ intersects the pre-image $\pi^{-1}\left(\mathcal{S}_{o}\right)$ in a single point. So, the previous argument cannot be used to infer that the deformation tensor vanishes identically. On the other hand, the same argument in 
Prop. 4.2 (iv) in 24] implies that the deformation tensor $\phi$ is bounded in the $d d^{c} \tau$-norm along each leaf $L$. By holomorphicity of the component of $\left.\phi\right|_{L}$ and Liouville Theorem, this implies that the components of $\left.\phi\right|_{L}$ are constant along each leaf, hence identically vanishing if $\varphi$ satisfies (4.2) at the tangent spaces of $\pi^{-1}\left(\mathcal{S}_{o}\right)$.

We have now all ingredients to prove the three claims of the theorem. Let us start with (ii). In this case, for each model $\left(\mathcal{M}_{o}(c), \tau\right)$ the Remmert reduction $\pi: M_{o}(c) \rightarrow \mathcal{M}_{o}(c)$ is the identity map and the assumption that $\varphi$ satisfies (4.2) at the tangent spaces of $\pi^{-1}\left(\mathcal{S}_{o}\right)$ coincides with the condition that $\varphi$ is a biholomorphism between the souls. By the above discussion, this occurs if and only if $\varphi$ is a biholomorphism, proving that $\left(\mathcal{M}_{o}(c), \tau\right)$ is soul rigid.

For the models considered in (i) and (iii), by the counterexamples in 4.3 .2 we know that none of them is soul rigid. Nonetheless, the above discussion shows that when $M$ is of mixed type or it has two ends and $c=+\infty$, if $\varphi$ is a biholomorphism at the blow ups of the souls, then it is a biholomorphism. This shows that in those cases, the model $\mathcal{M}_{o}(c)$ is soul semi-rigid, proving (iii) and the first claim of (i). The second claim in (i) is a consequence of the discussion at the end of 4.3 .2 .

\section{REFERENCES}

[1] H. Azad, A. Huckleberry and W. Richthofer, Homogeneous CR manifolds, J. Reine und Angew. Math. 358 (1985), 125-154.

[2] D. V. Alekseevsky and A. Spiro, Flag manifolds and homogeneous CR structures in "Recent advances in Lie theory (Vigo, 2000)", p. 3-44, Res. Exp. Math., 25, Heldermann, Lemgo, 2002.

[3] D. V. Alekseevsky and A. Spiro, Compact homogeneous CR manifolds, J. Geom. Anal. 12 (2002), 183-201.

[4] D.V. Alekseevsky and A. Spiro, Invariant CR structures on compact homogeneous manifolds, Hokkaido Math. J. 32 (2003), 209-276.

[5] J. Bland and T. Duchamp, Moduli for pointed convex domains, Invent. Math. 104 (1991), 61-112.

[7] G. E. Bredon, Introduction to compact transformation groups, Acad. Press, New York and London, 1972.

[8] D. Burns, Curvatures of Monge-Ampère foliations and parabolic manifolds, Ann. of Math. 115 (1982), 349-373.

[9] H. Cartan, Quotients of complex analytic spaces, In "Contrib. Funct. Theor.", Int. Collog. Bombay, 1960, 1-15.

[10] T. Duchamp and M. Kalka, Singular Monge-Ampere Foliations, Math. Ann. 325 (2003), 187-209.

[11] P. Griffiths and J. King, Nevanlinna Theory and Holomorphic Mappings bween Affine Varieties, Acta Math. 130 (1973), 145-220.

[12] V. V. Gorbatsevic and A. L. Onishchik, Lie Transformation Groups in "Encyclopoedia of Mathematical Sciences - Lie Groups and Lie Algebras I", ed A. L. Onishchik and E. B. Vinberg, Springer-Verlag VINITI, Berlin 1993 (Russian edition: VINITI, Moscow,1988)

[13] V.Guillelmin and M. Stenzel, Grauert tubes and the homogeneous Monge-Ampère equation, J. Diff. Geom. 34 (1991), 561-570.

[14] A. Huckleberry and E. Oeljeklaus, Classification theorems for almost homogeneous spaces, Institut Élie Cartan, 9. Université de Nancy, Institut Élie Cartan, Nancy, 1984.

[15] A. Huckleberry and D. Snow, Almost-homogeneous Kähler manifolds with hypersurface orbits, Osaka J. Math. 19 (1982), 763-786.

[16] L. Lempert, La métrique de Kobayashi et la représentation des domaines sur la boule, Bull. Soc. Math. France, 109 (1981), 427-474.

[17] L. Lempert, Holomorphic invariants, normal forms and the moduli space of convex domains, Ann. of Math. 128 (1988), 43-78.

[18] L. Lempert and R. Szöke, Global solutions of the homogeneous complex Monge-Ampère equation and complex structures on the tangent bundle of Riemannian manifolds, Math. Ann. 290 (1991), 689-712. 
[19] A. Morimoto and T. Nagano, On pseudo-conformal transformations of hypersurfaces, J. Math. Soc. Japan 15 (1963), 289-300.

[20] I. V. Mykytyuk, Invariant Kähler structures on the cotangent bundles of compact symmetric spaces, Nagoya Math. J. 169 (2003), 191-217.

[21] R. Narasimhan, The Levi Problem for Complex Spaces II, Math. Ann. 146 (1962), 195-216.

[22] G. Patrizio, Parabolic Exhaustions for Strictly Convex Domains, Manuscripta Math. 47 (1984), 271309.

[23] G. Patrizio, A characterization of complex manifolds biholomorphic to a circular domain, Math. Z. 189 (1985), 343-363.

[24] G. Patrizio and A. Spiro, Monge-Ampère equations and moduli spaces of manifolds of circular type, Adv. Math. 223 (2010), 174-197.

[25] G. Patrizio and A. Spiro, Pluripotential Theory and Monge-Ampère foliation in "Pluripotential theory, 265319, Lecture Notes in Math., 2075", Springer, Heidelberg, 2013.

[26] G. Patrizio and A. Spiro, Modular data and regularity of Monge-Ampre exhaustions and of Kobayashi distance, Math. Ann. 362 (2015), 425-449.

[27] G. Patrizio and P.-M. Wong, Stein manifolds with compact symmetric center, Math. Ann. 289 (1991), 355-382.

[28] F. Podestà and A. Spiro, Introduzione ai Gruppi di Transformazione, Volume of the Preprint Series of the Mathematics Department "V. Voleterra" of the University of Ancona, Via delle Brecce Bianche, Ancona, ITALY, 1996.

[29] F. Podestà and A. Spiro, New examples of almost homogeneous Kähler-Einstein manifolds, Indiana Univ. Math. J. 52 (2003), 1027-1074.

[30] R. Remmert, Sur les espaces analytiques holomorphiquement séparable et holomorphiquement convexes, C. R. Acad. Sci., Paris 243 (1956), 118-121.

[31] A. Spiro, The Ricci tensor of an almost homogeneous Kähler manifold, Adv. Geom. 3 (2003), $387-422$.

[32] M. Stenzel, Kähler Structures on Cotangent Bundles of Real Analytic Riemannian Manifolds, Ph. D. Thesis, Massachusetts Institute of Technology, Cambridge MA, 1990.

[33] W. Stoll, Value Distribution on Parabolic Spaces, Lecture Notes in Math. 600, Springer Verlag, 1977.

[34] W. Stoll, The characterization of strictly parabolic manifolds, Ann. Scuola Norm. Sup. Pisa, Cl. Sci. (4) 7 (1980), 87-154.

[35] W. Stoll, The characterization of strictly parabolic Spaces, Compositio Math 44 (1981), 305-373.

[36] P.-M. Wong, On Umbilical Hypersurfaces and Uniformization of Circular Domains, In "Complex Analysis of several Variables", p. 225-252, Proc. Symp. Pure Math. 41, A.M.S., Providence, 1984.

MORRIS KALKA

Mathematics Department

TUlane University

6823 St. Charles Ave.

New OrLeANS, LA 70118

USA

E-mail: kalka@math.tulane.edu
Giorgio Patrizio

Dip. Matematica e Informatica

"U. Dini"

Università di Firenze

\& Istituto Nazionale Di

Alta Matematica

"Francesco Severi"

E-mail: patrizio@math.unifi.it
Andrea Spiro

Scuola di Scienze e Tecnologie

Università di CAMERINO

Via Madonna delle Carceri

I-62032 CAmerino (Macerata)

ITALY

E-mail: andrea.spiro@unicam.it 